\title{
Investigations into the Role of Ion Pairing In the Reactions of Heteroatom- Substituted Cyclic Oxocarbenium Ions
}

\author{
Siddhartha R. Shenoy and K. A. Woerpel* \\ Department of Chemistry, University of California \\ Irvine, CA 92697-2025
}

\section{Supporting Information}

\section{Contents:}

I. Synthesis of the Ester Substrates $\quad S-1$

II. Methylenation of the Ester Substrates $\quad S-3$

III. Nucleophilic Substitutions of Acetate Substrates $\quad S-5$

IV. Lewis Acid-Mediated Rearrangement Reactions of Vinyl Acetals $\quad S-6$

V. Stereochemical Proofs of Nucleophilic Substituted Products and Rearrangement Products $\quad S-8$

VI. Bibliography S S-9

VII. Analytical Data $\quad S-10$

General. ${ }^{1} \mathrm{H}$ NMR and ${ }^{13} \mathrm{C}$ NMR spectra were recorded at ambient temperature at $500 \mathrm{MHz}$ and $100 \mathrm{MHz}$ and $125 \mathrm{MHz}$, respectively, using a Bruker DRX 400 or DRX 500 spectrometers. The data are reported as follows: chemical shift in ppm from internal tetramethylsilane on the $\delta$ scale, multiplicity $(\mathrm{br}=$ broad, $\mathrm{s}=$ singlet, $\mathrm{d}=$ doublet, $\mathrm{t}=$ triplet, $\mathrm{q}=$ quartet, $\mathrm{m}=$ multiplet), coupling constants $(\mathrm{Hz})$, and integration. Infrared (IR) spectra were obtained using a MIDAC Prospect FT-IR spectrometer. High resolution mass spectra were acquired on a VG Analytical 7070E or Fisons Autospec spectrometer, and were obtained by peak matching. Microanalyses were performed by Atlantic Microlab, Atlanta, GA. Analytical gas-liquid chromatography (GC) analyses were performed on a Hewlett Packard 5890 Level 4 Chromatograph, equipped with a split-mode injection system and a flame ionization detector. A fused silica capillary column $(30 \mathrm{~m} \times 0.32 \mathrm{~mm})$ wall-coated with DB-1 (J \& W Scientific) was used with helium as the carrier gas (16 psi column head pressure). Liquid chromatography was performed using forced flow (flash chromatography) of the indicated solvent system on EM Reagents silica gel $\left(\mathrm{SiO}_{2}\right) 60$ (230-400 mesh). All reactions were carried out under an atmosphere of nitrogen in glassware that had been flame-dried under vacuum $(0.05 \mathrm{mmHg})$. Unless otherwise noted, all reagents were commercially obtained and, where appropriate, purified prior to use. THF, $\mathrm{Et}_{2} \mathrm{O}$, and $\mathrm{CH}_{2} \mathrm{Cl}_{2}$ were dried by filtration through alumina. ${ }^{1}$

\section{Synthesis of the Ester Substrates}

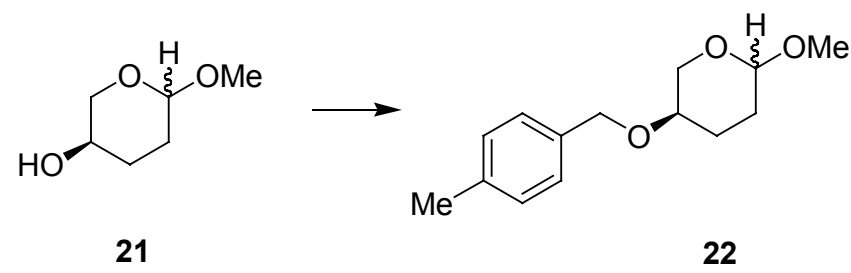

4-(4-Methylbenzyloxy)-1-methoxytetrahydropyran (22). To the neat alcohol 21 (2.6 g, $20 \mathrm{mmol})$ was added 4-methylbenzyl bromide $(8.1 \mathrm{~g}, 44 \mathrm{mmol})$ and finely ground $\mathrm{KOH}(4.5 \mathrm{~g}, 80 \mathrm{mmol}) .{ }^{2}$ The mixture was dissolved in THF $(30 \mathrm{~mL})$. The solution was stirred at $70{ }^{\circ} \mathrm{C}$ for $30 \mathrm{~h}$. The reaction mixture was cooled to 
$25{ }^{\circ} \mathrm{C}$ and filtered, and the filtrate was concentrated in vacuo to afford an orange oil. The orange oil was purified by column chromatography (30:70 - 60:40 EtOAc/hexanes) to afford the product (as a 3.2:1 mixture of anomers) as a colorless oil $(4.3 \mathrm{~g}, 93 \%) .{ }^{3}$ The purified product was characterized as a mixture of diastereomers: ${ }^{1} \mathrm{H}$ NMR $\left(500 \mathrm{MHz}, \mathrm{CDCl}_{3}\right) \delta$ 7.26-7.12 (m, 5.7H), 4.57-4.51 (m, 4H), $3.86(\mathrm{dd}, J=11.9,2.3,1 \mathrm{H}), 3.65$ (ddd, $J=10.9,4.5,1.8,0.3 \mathrm{H}), 3.61-3.53(\mathrm{~m}, 1.3 \mathrm{H}), 3.51-3.42(\mathrm{~m}, 1.4 \mathrm{H}), 3.40(\mathrm{~s}, 3 \mathrm{H}), 3.36(\mathrm{~s}, 0.9 \mathrm{H}), 2.34(\mathrm{~s}, 3.9 \mathrm{H})$, $2.10-1.95(\mathrm{~m}, 2.0 \mathrm{H}), 1.90-1.80(\mathrm{~m}, 0.9 \mathrm{H}), 1.70-1.60(\mathrm{~m}, 1.3 \mathrm{H}), 1.50(\mathrm{~m}, 0.9 \mathrm{H}) ;{ }^{13} \mathrm{C} \mathrm{NMR}\left(125 \mathrm{MHz}, \mathrm{CDCl}_{3}\right) \delta$ $137.8,137.7,136.0,129.5,128.2,128.1,99.9,98.2,72.7,71.6,70.8,70.7,63.7,63.1,55.7,55.1,29.0,26.8$, 25.4, 24.1, 21.6; IR (thin film) 2933, 2866, 1216, 1132, 1091, $1040 \mathrm{~cm}^{-1}$; HRMS (GC-MS, EI) $\mathrm{m} / \mathrm{z}$ calcd for $\mathrm{C}_{14} \mathrm{H}_{21} \mathrm{O}_{3}(\mathrm{M}+\mathrm{H})^{+}$237.1491, found 237.1490. Anal. Calcd for $\mathrm{C}_{14} \mathrm{H}_{20} \mathrm{O}_{3}$ : C, 71.16; $\mathrm{H}, 8.53$. Found: C, 71.10; $\mathrm{H}, 8.52$.

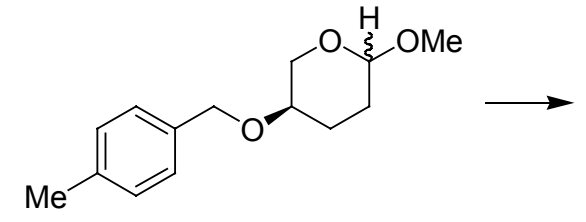

22

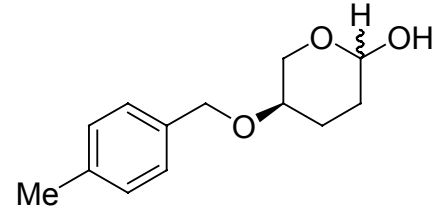

23

4-(4-Methylbenzyloxy)-1-hydroxytetrahydropyran (23). A mixture of 22 (2.70 g, $11.3 \mathrm{mmol})$ and 50:50 AcOH: $\mathrm{H}_{2} \mathrm{O}(100 \mathrm{~mL})$ was stirred at $100{ }^{\circ} \mathrm{C}$ for $18 \mathrm{~h}$. The solvent was removed in vacuo as an azeotrope with toluene $(4 \times 50 \mathrm{~mL})$. The resulting yellow oil was purified by column chromatography $(10: 90-30: 70$ EtOAc/hexanes) to afford the product (as a 1:1 mixture of anomers) as a colorless oil $(2.2 \mathrm{~g}, 88 \%){ }^{3} \quad$ The purified product was characterized as a mixture of diastereomers: ${ }^{1} \mathrm{H}$ NMR $\left(500 \mathrm{MHz}, \mathrm{CDCl}_{3}\right) \delta 7.26-7.15(\mathrm{~m}$, $8 \mathrm{H}), 4.97(\mathrm{~m}, 2 \mathrm{H}), 4.52(\mathrm{~m}, 4.3 \mathrm{H}), 4.05(\mathrm{~m}, 1 \mathrm{H}), 3.90(\mathrm{dd}, J=11.4,7.0,1 \mathrm{H}), 3.84(\mathrm{~d}, J=4.5,1 \mathrm{H}), 3.77(\mathrm{dd}, J=$ 4.3, 0.9, 1H), 3.62 (ddd, $J=11.4,3.5,0.9,1 \mathrm{H}), 3.50-3.40(\mathrm{~m}, 3 \mathrm{H}), 2.34(\mathrm{~s}, 6 \mathrm{H}), 2.08-1.99(\mathrm{~m}, 2 \mathrm{H}), 1.92-1.91$ $(\mathrm{m}, 1 \mathrm{H}), 1.84-1.78(\mathrm{~m}, 2 \mathrm{H}), 1.68-1.61(\mathrm{~m}, 2 \mathrm{H}), 1.50-1.47(\mathrm{~m}, 1 \mathrm{H}) ;{ }^{13} \mathrm{C} \mathrm{NMR}\left(125 \mathrm{MHz}, \mathrm{CDCl}_{3}\right) \delta 137.8$, 137.7, 135.9, 135.8, 129.6, 129.5, 128.2, 94.3, 93.1, 71.8, 71.7, 70.9, 70.6, 65.3, 64.7, 29.3, 28.6, 25.5, 25.2, 21.6; IR (thin film) 3649, 3155, 2954, 2872, $2254 \mathrm{~cm}^{-1}$; HRMS (GC-MS, EI) $m / z$ calcd for $\mathrm{C}_{13} \mathrm{H}_{17} \mathrm{O}_{3}(\mathrm{M}-\mathrm{H})^{+}$ 221.1178 , found 221.1178 .

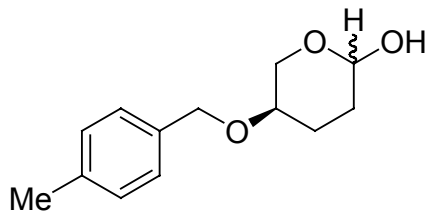

23

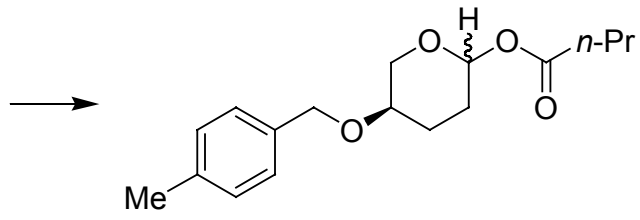

24

4-(4-Methylbenzyloxy)tetrahydropyran-1-butyrate ester (24). To a cooled $\left(0{ }^{\circ} \mathrm{C}\right)$ solution of hemiacetal 23 in $\mathrm{CH}_{2} \mathrm{Cl}_{2}$ (45 mL) was added DMAP (0.40 g, $\left.3.3 \mathrm{mmol}\right), \mathrm{Et}_{3} \mathrm{~N}$ (1.8 mL, $\left.13 \mathrm{mmol}\right)$, and butyric anhydride $(1.2 \mathrm{~mL}, 7.3 \mathrm{mmol})$. The reaction mixture was warmed to $25{ }^{\circ} \mathrm{C}$ and stirred for $2 \mathrm{~h}$. At this time the mixture was concentrated in vacuo to afford a yellow residue. Purification by column chromatography $(0: 100-$ 30:70 EtOAc/hexanes) afforded the product (as a 10:7 trans:cis mixture of anomers) as a clear oil (1.3 g, 99\%). ${ }^{3}$ The purified product was characterized as a mixture of diastereomers: ${ }^{1} \mathrm{H}$ NMR $\left(500 \mathrm{MHz}, \mathrm{CDCl}_{3}\right) \delta 7.26-7.14$ $(\mathrm{m}, 6.8 \mathrm{H}), 6.03(\mathrm{t}, J=3.3,1 \mathrm{H}), 5.99(\mathrm{dd}, J=2.8,2.4,0.7 \mathrm{H}), 4.55-4.53(\mathrm{~m}, 3.4 \mathrm{H}), 3.91(\mathrm{dd}, J=12.2,2.4,1 \mathrm{H})$, $3.80(\mathrm{ddd}, J=10.8,4.4,1.8,0.7 \mathrm{H}), 3.74(\mathrm{ddd}, J=12.2,3.7,1.8,1 \mathrm{H}), 3.64(\mathrm{t}, J=9.4,0.7 \mathrm{H}), 3.53-3.50(\mathrm{~m}$, $1.7 \mathrm{H}), 2.34-2.31(\mathrm{~m}, 8.5 \mathrm{H}) 2.22-2.13(\mathrm{~m}, 0.7 \mathrm{H}), 2.05-1.98(\mathrm{~m}, 1.7 \mathrm{H}), 1.81-1.78(\mathrm{~m}, 3.4 \mathrm{H}), 1.68(\mathrm{dqd}, J=$ $14.8,7.4,1.9,3.4 \mathrm{H}), 1.60-1.58(\mathrm{~m}, 1 \mathrm{H}), 0.96(\mathrm{t}, J=7.4,5 \mathrm{H}) ;{ }^{13} \mathrm{C} \mathrm{NMR}\left(125 \mathrm{MHz}, \mathrm{CDCl}_{3}\right) \delta 172.7,137.8$, 
$135.7,129.60,129.55,128.20,128.16,92.6,91.1,71.9,71.0,70.8,70.7,64.90,64.86,36.8,28.0,25.3,25.1$, 23.4, 21.6, 18.8, 14.1; IR (thin film) 2947, 2876, 1741, 1175, 1084, $1036 \mathrm{~cm}^{-1}$; HRMS (GC-MS, EI) $\mathrm{m} / z$ calcd for $\mathrm{C}_{17} \mathrm{H}_{24} \mathrm{O}_{4}(\mathrm{M}+\mathrm{Na})^{+}$315.1572, found 315.1836. Anal. Calcd for $\mathrm{C}_{17} \mathrm{H}_{24} \mathrm{O}_{4}$ : C, 69.84; H, 8.27. Found: $\mathrm{C}$, $69.61 ; \mathrm{H}, 8.04$.

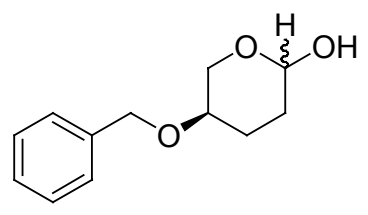

25

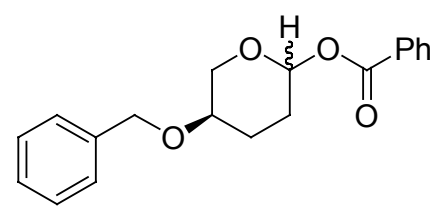

26

4-Benzyloxytetrahydropyran-1-benzoate ester (26). To a cooled $\left(0{ }^{\circ} \mathrm{C}\right)$ solution of hemiacetal $\mathbf{2 5}^{2}$ (0.18 g, $0.86 \mathrm{mmol})$ in $\mathrm{CH}_{2} \mathrm{Cl}_{2}(10 \mathrm{~mL})$ was added DMAP $(0.029 \mathrm{~g}, 0.28 \mathrm{mmol}), \mathrm{Et}_{3} \mathrm{~N}(0.30 \mathrm{~mL}, 2.2 \mathrm{mmol})$, and benzoyl chloride $(0.12 \mathrm{~mL}, 1.0 \mathrm{mmol})$. The reaction mixture was warmed to $25^{\circ} \mathrm{C}$ and stirred for $2 \mathrm{~h}$. The reaction mixture was then concentrated in vacuo to afford a yellow residue. Purification by column chromatography (0:100 - 20:80 EtOAc/hexanes) afforded the product (as a 1:1 mixture of anomers) as a clear oil $(0.25 \mathrm{~g}, 92 \%) .{ }^{3}$ The purified product was characterized as a mixture of diastereomers: ${ }^{1} \mathrm{H}$ NMR $(400 \mathrm{MHz}$, $\left.\mathrm{CDCl}_{3}\right) \delta 8.09-8.06(\mathrm{~m}, 4 \mathrm{H}), 7.58-7.29(\mathrm{~m}, 16 \mathrm{H}), 6.31(\mathrm{t}, J=3.0,1 \mathrm{H}), 6.26(\mathrm{dd}, J=2.8,3.1,1 \mathrm{H}), 4.65-4.57$ $(\mathrm{m}, 4 \mathrm{H}), 4.03(\mathrm{dd}, J=2.1,12.3,1 \mathrm{H}), 3.88-3.86(\mathrm{~m}, 2 \mathrm{H}), 3.77(\mathrm{dd}, J=10.9,9.7,1 \mathrm{H}), 3.67-3.56(\mathrm{~m}, 2 \mathrm{H}), 2.38$ $2.26(\mathrm{~m}, 1 \mathrm{H}), 2.18-2.03(\mathrm{~m}, 3 \mathrm{H}), 1.22-1.84(\mathrm{~m}, 3 \mathrm{H}), 1.77(\mathrm{dtd}, J=13.7,4.3,3.2,1 \mathrm{H}) ;{ }^{13} \mathrm{C}$ NMR $(125 \mathrm{MHz}$, $\left.\mathrm{CDCl}_{3}\right) \delta 165.6,165.5,138.8,138.7,133.69,133.66,130.7,130.5,130.3,130.2,129.0,128.91,128.89,128.3$, $128.2,128.1,128.0,93.3,91.8,72.3,71.3,71.0,70.8,65.1,64.7,28.3,25.4,25.0,23.2$; IR (thin film) 2254 , 1720, 1453, 1381, 1273, 1087, $910 \mathrm{~cm}^{-1}$; HRMS (GC-MS, EI) $\mathrm{m} / \mathrm{z}$ calcd for $\mathrm{C}_{19} \mathrm{H}_{20} \mathrm{O}_{4}(\mathrm{M}+\mathrm{Na})^{+} 335.1259$, found 335.1245. Anal. Calcd for $\mathrm{C}_{19} \mathrm{H}_{20} \mathrm{O}_{4}$ : C, 73.06; H, 6.45. Found: $\mathrm{C}, 72.79 ; \mathrm{H}, 6.32$.

\section{Methylenation of the Ester Substrates}

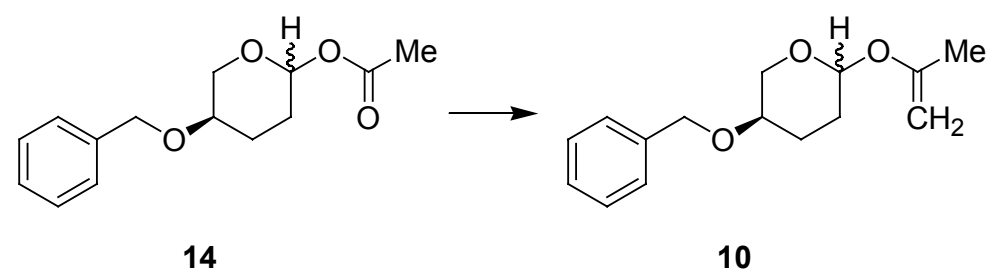

4-Benzyloxytetrahydropyran-1-(1'-methylvinyl) acetal (10). To neat acetate $14(0.20 \mathrm{~g}, 0.68 \mathrm{mmol})$ was added $\mathrm{Cp}_{2} \mathrm{TiMe}_{2}(4.8 \mathrm{~mL}, 0.5 \mathrm{M}$ solution in THF, $2.4 \mathrm{mmol}){ }^{4}$. The orange solution was protected from light and heated at $65{ }^{\circ} \mathrm{C}$ for 3 days. The reaction mixture was then cooled to $25^{\circ} \mathrm{C}$, diluted with $\mathrm{Et}_{2} \mathrm{O}(10 \mathrm{~mL})$, and filtered through Celite 521. The filtrate was concentrated in vacuo to afford an orange oil. Purification by column chromatography (0:100 - 30:70 EtOAc/hexanes) afforded the product (as a 5:4 mixture of anomers) as a clear oil $(0.08 \mathrm{~g}, 44 \%)$. The purified product was characterized as a mixture of diastereomers: ${ }^{1} \mathrm{H}$ NMR (500 $\left.\mathrm{MHz}, \mathrm{CDCl}_{3}\right) \delta 7.37-7.26(\mathrm{~m}, 9 \mathrm{H}), 5.25(\mathrm{dd}, J=3.4,3.0,0.8 \mathrm{H}), 5.22(\mathrm{t}, J=2.8,1 \mathrm{H}), 4.62-4.55(\mathrm{~m}, 3.6 \mathrm{H}), 4.21$ $(\mathrm{d}, J=1.3,0.8 \mathrm{H}), 4.18(\mathrm{~d}, J=1.3,1 \mathrm{H}), 4.03-4.02(\mathrm{~m}, 1.8 \mathrm{H}), 3.89(\mathrm{dd}, J=12.0,2.42,0.8 \mathrm{H}), 3.71(\mathrm{ddd}, J=$ $10.8,4.5,1.7,1 \mathrm{H}), 3.67-3.59(\mathrm{~m}, 1.8 \mathrm{H}), 3.56-3.47(\mathrm{~m}, 1.8 \mathrm{H}), 2.17-2.03(\mathrm{~m}, 1.8 \mathrm{H}), 2.01-1.85(\mathrm{~m}, 2.8 \mathrm{H}), 1.83-$ $1.82(\mathrm{~m}, 5.4 \mathrm{H}), 1.81-1.73(\mathrm{~m}, 0.8 \mathrm{H}), 1.72-1.57(\mathrm{~m}, 1.8 \mathrm{H}) ;{ }^{13} \mathrm{C} \mathrm{NMR}\left(125 \mathrm{MHz}, \mathrm{CDCl}_{3}\right) \delta 157.4,157.2,138.8$, 138.7, 128.70, 128.67, 128.0, 127.9, 127.8, 95.0, 93.5, 86.0, 85.8, 72.5, 71.3, 70.9, 70.6, 63.6, 63.4, 28.7, 26.0, 
25.2, 23.6, 21.1; IR (thin film) 1355, 1083, 734, $699 \mathrm{~cm}^{-1}$; HRMS (GC-MS, EI) $\mathrm{m} / \mathrm{z}$ calcd for $\mathrm{C}_{15} \mathrm{H}_{20} \mathrm{O}_{3}$ $(\mathrm{M}+\mathrm{Na})^{+} 271.1310$, found 271.1309 .

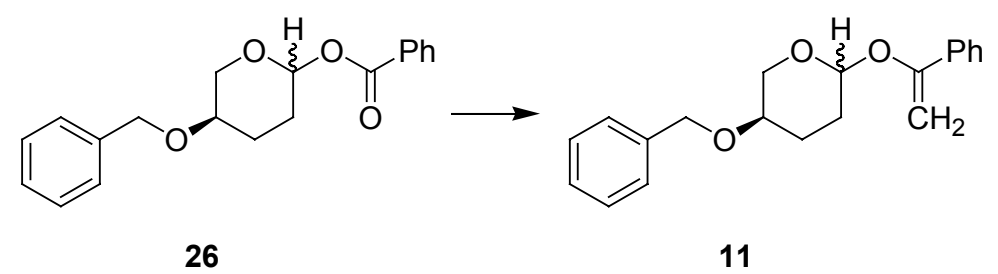

4-Benzyloxytetrahydropyran-1-(1'-phenylvinyl) acetal (11). To neat benzoate 26 ( $0.12 \mathrm{~g}, 0.4 \mathrm{mmol})$ was added $\mathrm{Cp}_{2} \mathrm{TiMe}_{2}(4.0 \mathrm{~mL}, 0.5 \mathrm{M}$ solution in THF, $2.0 \mathrm{mmol}) .{ }^{4}$ The orange solution was protected from light and heated at $65{ }^{\circ} \mathrm{C}$ for 3 days. The reaction mixture was then cooled to $25{ }^{\circ} \mathrm{C}$, diluted with pentane $(10$ $\mathrm{mL}$ ), and filtered through Celite 521. The filtrate was concentrated in vacuo to afford an orange oil. Purification by column chromatography $\left(0: 100-10: 90 \mathrm{Et}_{2} \mathrm{O} /\right.$ pentane) afforded the product (as a 3:1 mixture of anomers) as a clear oil $(0.08 \mathrm{~g}, 72 \%)$. The purified product was characterized as a mixture of diastereomers: ${ }^{1} \mathrm{H}$ NMR (500 MHz, $\left.\mathrm{CDCl}_{3}\right) \delta 7.68-7.65(\mathrm{~m}, 2.6 \mathrm{H}), 7.40-7.31(\mathrm{~m}, 10.4 \mathrm{H}), 5.51(\mathrm{t}, J=2.9,0.3 \mathrm{H}), 5.44(\mathrm{dd}, J=$ 2.6, 2.3, 1H), $4.87(\mathrm{~d}, J=2.5,1.3 \mathrm{H}), 4.70(\mathrm{~d}, J=2.4,0.3 \mathrm{H}), 4.66-4.55(\mathrm{~m}, 3.6 \mathrm{H}), 3.96(\mathrm{dd}, J=12.3,2.1,0.3 \mathrm{H})$, $3.84-3.75(\mathrm{~m}, 1.3 \mathrm{H}), 3.71(\mathrm{dd}, J=10.7,9.6,1 \mathrm{H}), 3.62(\mathrm{tt}, J=9.5,4.5,1 \mathrm{H}), 3.55(\mathrm{~m}, 0.3 \mathrm{H}), 2.35-1.99(\mathrm{~m}$, 3.6H), 1.98-1.90 (m, 0.3H), 1.90-1.80 (m, 1.3H); ${ }^{13} \mathrm{C}$ NMR (125 MHz, $\left.\mathrm{CDCl}_{3}\right) \delta 157.3,138.6,136.4,128.64$, $128.61,128.58,128.3,127.81,127.78,125.5,95.5,94.1,87.0,86.8,72.5,71.1,70.8,70.5,63.4,63.1,29.0$, 25.7, 25.3, 23.0; IR (thin film) 3084, 3060, 1621, 1574, 1494, 1298, $1280 \mathrm{~cm}^{-1}$.

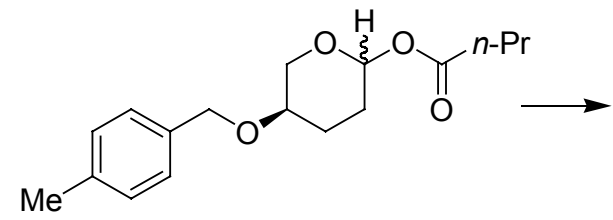

24

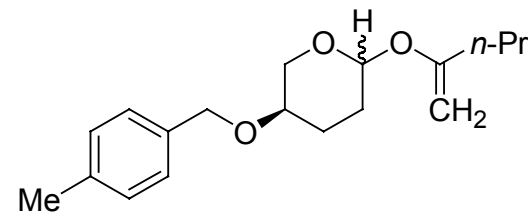

17

4-(4-Methylbenzyloxy)tetrahydropyran-1-(1'-n-propylvinyl) acetal (17). To neat butyrate $24(0.53$ $\mathrm{g}, 1.8 \mathrm{mmol})$ was added $\mathrm{Cp}_{2} \mathrm{TiMe}_{2}(10 \mathrm{~mL}, 0.5 \mathrm{M}$ solution in THF, $5.0 \mathrm{mmol}){ }^{4}$ The orange solution was protected from light and heated at $65{ }^{\circ} \mathrm{C}$ for 3 days. At this time, the reaction mixture was cooled to $25{ }^{\circ} \mathrm{C}$, diluted with pentane $(10 \mathrm{~mL})$ and filtered through Celite 521 . The filtrate was concentrated in vacuo to afford an orange oil. Purification by column chromatography (pentane - 2:98 acetone/pentane) afforded the product (as a 2:1 trans:cis mixture of anomers) as a clear oil $(0.24 \mathrm{~g}, 47 \%)$. The purified product was characterized as a mixture of diastereomers: ${ }^{1} \mathrm{H}$ NMR $\left(500 \mathrm{MHz}, \mathrm{CDCl}_{3}\right) \delta 7.25-7.14(\mathrm{~m}, 6 \mathrm{H}), 5.24(\mathrm{t}, J=3.2,1 \mathrm{H}), 5.21(\mathrm{~m}$, $0.5 \mathrm{H}), 4.60-4.47(\mathrm{~m}, 3 \mathrm{H}), 4.22(\mathrm{~d}, J=1.5,1 \mathrm{H}), 4.18(\mathrm{~d}, J=1.5,0.5 \mathrm{H}), 4.00(\mathrm{~m}, 1.5 \mathrm{H}), 3.87(\mathrm{dd}, J=12.1,2.3$, $1 \mathrm{H}$ ), 3.69 (ddd, $J=10.7,4.4,2.0,0.5 \mathrm{H}), 3.64$ (ddd, $J=12.1,3.7,1.8,1 \mathrm{H}), 3.57$ (dd, $J=10.4,9.7,0.5 \mathrm{H}$ ), 3.54$3.45(\mathrm{~m}, 1.5 \mathrm{H}), 2.34(\mathrm{~s}, 4.5 \mathrm{H}), 2.17-2.00(\mathrm{~m}, 6.5 \mathrm{H}), 1.99-1.83(\mathrm{~m}, 2 \mathrm{H}), 1.82-1.66(\mathrm{~m}, 2 \mathrm{H}), 1.64-1.58(\mathrm{~m}, 1 \mathrm{H})$, $1.55-1.46(\mathrm{~m}, 3 \mathrm{H}), 0.92(\mathrm{t}, J=7.4,4.5 \mathrm{H}) ;{ }^{13} \mathrm{C}$ NMR $\left(125 \mathrm{MHz}, \mathrm{CDCl}_{3}\right) \delta 161.0,160.8,137.7,135.9,129.6$, $129.5,128.2,128.1,95.1,93.5,85.2,85.0,72.5,71.2,70.9,70.6,63.7,63.5,37.5,37.4,29.0,26.1,25.4,23.7$, 21.6, 21.0, 14.1; IR (thin film) 2931, 2924, 2862, 2856, 1516, 1352, 1083, $805 \mathrm{~cm}^{-1}$, HRMS (GC-MS, EI) $\mathrm{m} / \mathrm{z}$ calcd for $\mathrm{C}_{18} \mathrm{H}_{26} \mathrm{O}_{3}(\mathrm{M}+\mathrm{Na})^{+}$313.1780, found 313.1776. Anal. Calcd for $\mathrm{C}_{18} \mathrm{H}_{26} \mathrm{O}_{3}: \mathrm{C}, 74.45 ; \mathrm{H}, 9.02$. Found: C, $74.43 ; \mathrm{H}, 9.17$. 


\section{Nucleophilic Substitutions of Acetate Substrates}

General Procedure for Nucleophilic Substitutions of Acetate Substrates. Silylenol ether (4.0 equiv) was added to a cooled $\left(-78{ }^{\circ} \mathrm{C}\right)$ solution of acetate $\left(1.0\right.$ equiv) in PhMe $(0.15 \mathrm{M})$. After 2 min, $\mathrm{BF}_{3} \cdot \mathrm{OEt}_{2}(1.3$ equiv) was added dropwise by syringe, and the reaction mixture was warmed to $25{ }^{\circ} \mathrm{C}$. After $30 \mathrm{~min}$, the $\mathrm{Na}_{2} \mathrm{CO}_{3}(1 \mathrm{~mL}$ per mmol of acetate) was added to the reaction mixture and diluted with EtOAc (1 mL per mmol of acetate) and $\mathrm{H}_{2} \mathrm{O}(2 \mathrm{~mL}$ per mmol of acetate). The layers were separated and the aqueous layer was extracted four times with EtOAc ( $2 \mathrm{~mL}$ per mmol of acetate). The combined organic layers were washed once with brine ( $2 \mathrm{~mL}$ per mmol of acetate), dried over $\mathrm{Na}_{2} \mathrm{SO}_{4}$, filtered, and concentrated in vacuo. The diastereomeric ratio of products was determined by GC analysis of the unpurified product mixture and confirmed using ${ }^{1} \mathrm{H}$ NMR spectroscopy.

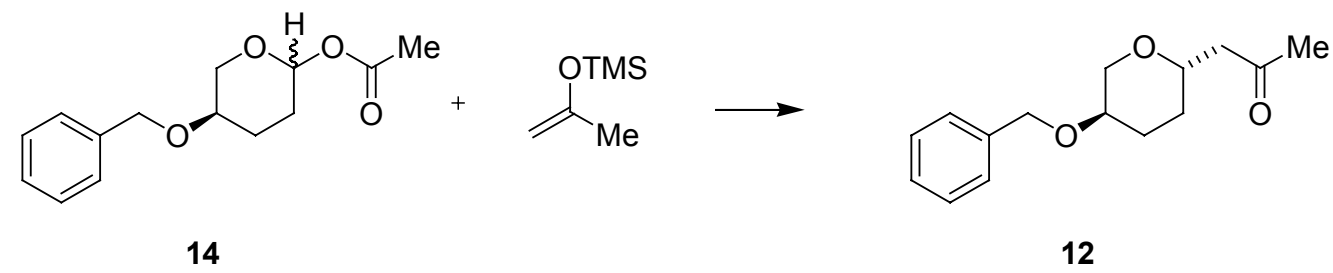

4-Benzyloxytetrahydropyran-1-(2'-methyl-2'-ethanone) (12). Using the general nucleophilic substitution conditions, acetate $14(0.11 \mathrm{~g}, 0.37 \mathrm{mmol})$ and 2-(trimethylsilyloxy)propene $(0.24 \mathrm{~mL}, 1.4 \mathrm{mmol})$ were treated with $\mathrm{BF}_{3} \cdot \mathrm{OEt}_{2}(56 \mu \mathrm{L}, 0.47 \mathrm{mmol})$ to afford the product $(0.08 \mathrm{~g}, 88 \%)$ as an $85: 15$ trans/cis mixture of isomers. The oil was purified by column chromatography $(0: 100-20: 80$ EtOAc/pentane $)$ : GC $\mathrm{t}_{\mathrm{R}}$ (major) $12.7 \mathrm{~min}, \mathrm{t}_{\mathrm{R}}$ (minor) $12.5 \mathrm{~min}\left(80{ }^{\circ} \mathrm{C}\right.$ for $\left.1 \mathrm{~min}, 10^{\circ} / \mathrm{min}\right) ;{ }^{1} \mathrm{H}$ NMR $\left(400 \mathrm{MHz}, \mathrm{CDCl}_{3}\right) \delta 7.36-7.26$ $(\mathrm{m}, 5 \mathrm{H}), 4.58(\mathrm{~d}, J=11.9,1 \mathrm{H}), 4.52(\mathrm{~d}, J=11.9,1 \mathrm{H}),(\mathrm{m}, 2 \mathrm{H}), 4.05$ (ddd, $J=10.8,4.7,2.3,1 \mathrm{H}), 3.71$ (dddd, $J$ $=9.9,7.4,4.9,2.4,1 \mathrm{H}), 3.44(\mathrm{tt}, J=10.3,4.6,1 \mathrm{H}), 3.19(\mathrm{t}, J=10.5,1 \mathrm{H}), 2.65(\mathrm{dd}, J=7.7,8.2,1 \mathrm{H}), 2.43(\mathrm{dd}, J$ $=4.9,15.8,1 \mathrm{H}), 2.22-2.15(\mathrm{~m}, 1 \mathrm{H}), 2.17(\mathrm{~s}, 3 \mathrm{H}), 1.78(\mathrm{ddd}, J=13.2,5.8,3.5,1 \mathrm{H}), 1.49(\mathrm{tdd}, J=13.2,10.9$, 4.1, 1H), $1.32(\mathrm{tdd}, J=13.2,11.0,3.7,1 \mathrm{H}) ;{ }^{13} \mathrm{C} \mathrm{NMR}\left(125 \mathrm{MHz}, \mathrm{CDCl}_{3}\right) \delta 207.4,138.9,128.9,128.1,128.0$, 77.2, 74.1, 73.2, 71.2, 50.2, 31.3, 30.9, 30.4; IR (thin film) 1713, 1272, 1072, 1028, $698 \mathrm{~cm}^{-1}$; HRMS (GC-MS, EI) $m / z$ calcd for $\mathrm{C}_{15} \mathrm{H}_{20} \mathrm{O}_{3}(\mathrm{M}+\mathrm{Na})^{+} 271.1310$, found 271.1318 .

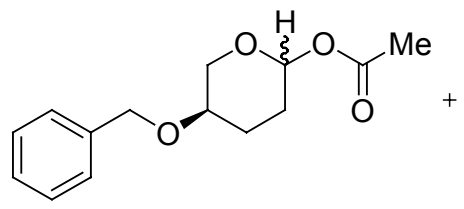

14

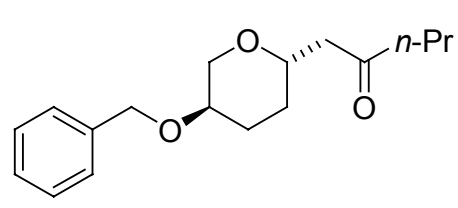

19

4-Benzyloxytetrahydropyran-1-(2'-n-propyl-2'-ethanone) (19). 2-(Trimethylsilyloxy)-1-pentene was prepared according to the literature procedure. ${ }^{5}$ Using the general nucleophilic substitution conditions, acetate $14(0.10 \mathrm{~g}, 0.41 \mathrm{mmol})$ and 2-(trimethylsilyloxy)-1-pentene $(0.2 \mathrm{~mL}, 1.0 \mathrm{mmol})$ were treated with $\mathrm{BF}_{3} \cdot \mathrm{OEt}_{2}$ $(0.09 \mathrm{~mL}, 0.70 \mathrm{mmol})$ to afford the product $(0.10 \mathrm{~g}, 92 \%)$ as an 80:20 trans $/$ cis mixture of isomers. The oil was purified by column chromatography $\left(0: 100-20: 80 \mathrm{EtOAc} /\right.$ pentane): $\mathrm{GC} \mathrm{t}_{\mathrm{R}}$ (major) $14.6 \mathrm{~min}, \mathrm{t}_{\mathrm{R}}$ (minor) 14.4 $\min \left(80{ }^{\circ} \mathrm{C}\right.$ for $\left.1 \mathrm{~min}, 10^{\circ} / \mathrm{min}\right) ;{ }^{1} \mathrm{H}$ NMR $\left(500 \mathrm{MHz}, \mathrm{CDCl}_{3}\right) \delta 7.35-7.26(\mathrm{~m}, 5 \mathrm{H}), 4.58(\mathrm{~d}, J=11.9,1 \mathrm{H}), 4.52$ $(\mathrm{d}, J=11.9,1 \mathrm{H}), 4.04(\mathrm{ddd}, J=10.8,4.7,2.3,1 \mathrm{H}), 3.73(\mathrm{dddd}, J=11.1,7.3,5.1,2.1,1 \mathrm{H}), 3.43(\mathrm{tt}, J=10.3$, $4.6,1 \mathrm{H}), 3.19(\mathrm{t}, J=10.5,1 \mathrm{H}), 2.63(\mathrm{dd}, J=15.8,7.6,1 \mathrm{H}), 2.45-2.35(\mathrm{~m}, 3 \mathrm{H}), 2.22-2.14(\mathrm{~m}, 1 \mathrm{H}), 1.78(\mathrm{ddd}, J$ 
$=13.3,6.1,3.6,1 \mathrm{H}), 1.59(\mathrm{dq}, J=14.8,7.4,2 \mathrm{H}), 1.49(\mathrm{tdd}, J=13.1,11.0,4.2,1 \mathrm{H}), 1.31(\operatorname{tdd}, J=13.4,11.1$, 3.8, 1H), $0.90(\mathrm{t}, J=7.4,3 \mathrm{H}) ;{ }^{13} \mathrm{C} \mathrm{NMR}\left(125 \mathrm{MHz}, \mathrm{CDCl}_{3}\right) \delta 209.5,138.9,128.9,128.1,128.0,74.2,73.1$, 71.2, 71.1, 49.0, 46.1, 30.9, 30.4, 17.4, 14.1; IR (thin film) 2987, 1737, 1374, 1235, $1046 \mathrm{~cm}^{-1} \mathrm{HRMS}$ (GC-MS, EI) $m / z$ calcd for $\mathrm{C}_{17} \mathrm{H}_{25} \mathrm{O}_{3}(\mathrm{M}+\mathrm{H})^{+}$277.1804, found 277.1802. Anal. Calcd for $\mathrm{C}_{17} \mathrm{H}_{24} \mathrm{O}_{3}: \mathrm{C}, 73.88 ; \mathrm{H}, 8.75$. Found: C, 73.82; H, 8.80.

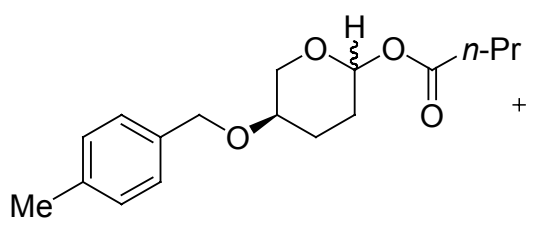

24
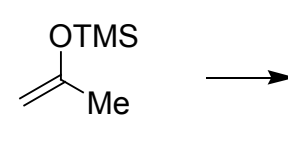

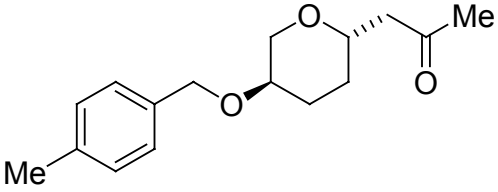

20

4-(4-Methylbenzyloxy)tetrahydropyran-1-(2'-methyl-2'-ethanone) (20). Using the general nucleophilic substitution conditions, ester $24(0.11 \mathrm{~g}, 0.37 \mathrm{mmol})$ and 2-(trimethylsilyloxy)propene $(0.24 \mathrm{~mL}$, $1.4 \mathrm{mmol})$ were treated with $\mathrm{BF}_{3} \cdot \mathrm{OEt}_{2}(0.06 \mathrm{~mL}, 0.43 \mathrm{mmol})$ to afford the product $(0.09 \mathrm{~g}, 88 \%)$ as an $80: 20$ trans/cis mixture of isomers. The oil was purified by column chromatography $(0: 100-20: 80 \mathrm{EtOAc} /$ pentane $)$ : $\mathrm{GC} \mathrm{t}_{\mathrm{R}}$ (major) $13.7 \mathrm{~min}, \mathrm{t}_{\mathrm{R}}$ (minor) $13.6 \mathrm{~min}\left(80{ }^{\circ} \mathrm{C}\right.$ for $\left.1 \mathrm{~min}, 10^{\circ} / \mathrm{min}\right) ;{ }^{1} \mathrm{H} \mathrm{NMR}\left(400 \mathrm{MHz}, \mathrm{CDCl}_{3}\right) \delta 7.21-$ $7.12(\mathrm{~m}, 4 \mathrm{H}), 4.54(\mathrm{~d}, J=11.7,1 \mathrm{H}), 4.47(\mathrm{~d}, J=11.7,1 \mathrm{H}), 4.02(\mathrm{ddd}, J=10.8,4.7,2.3,1 \mathrm{H}), 3.70(\mathrm{dddd}, J=$ 11.0, 7.4, 5.0, 2.1, 1H), $3.42(\mathrm{tt}, J=10.3,4.6,1 \mathrm{H}), 3.17(\mathrm{t}, J=10.5,1 \mathrm{H}), 2.63(\mathrm{dd}, J=15.8,7.7,1 \mathrm{H}), 2.42(\mathrm{dd}$, $J=15.8,4.9,1 \mathrm{H}), 2.33(\mathrm{~s}, 3 \mathrm{H}), 2.21-2.13(\mathrm{~m}, 4 \mathrm{H}), 1.76(\mathrm{ddd}, J=13.2,5.7,3.5,1 \mathrm{H}), 1.47(\mathrm{tdd}, J=13.2,10.9$, 4.1, $1 \mathrm{H}), 1.31$ (tdd, $J=13.3,10.9,3.7,1 \mathrm{H}) ;{ }^{13} \mathrm{C}$ NMR $\left(125 \mathrm{MHz}, \mathrm{CDCl}_{3}\right) \delta 207.4,137.9,135.9,129.6,128.2$, 74.1, 72.9, 71.2, 71.1, 49.9, 31.3, 30.9, 30.4, 21.6; IR (thin film) 2930, 2859, 1732, 1603, 1585, 1452, 1268, 1024, $709 \mathrm{~cm}^{-1}$ HRMS (GC-MS, EI) $\mathrm{m} / \mathrm{z}$ calcd for $\mathrm{C}_{16} \mathrm{H}_{22} \mathrm{O}_{3}(\mathrm{M}+\mathrm{Na})^{+}$285.1467, found 285.1466. Anal. Calcd for $\mathrm{C}_{16} \mathrm{H}_{22} \mathrm{O}_{3}$ : C, 73.25; $\mathrm{H}, 8.45$. Found $\mathrm{C}, 72.43 ; \mathrm{H}, 8.46$.

\section{Lewis Acid-Mediated Rearrangement Reactions of Vinyl Acetals}

Procedure A: General Procedure for Lewis Acid-Mediated Rearrangement of Vinyl Acetals. To a cooled $\left(-78{ }^{\circ} \mathrm{C}\right)$ solution of the vinyl acetal $(0.1-0.15 \mathrm{mmol})$ in dry solvent $(0.15 \mathrm{M})$ was added the Lewisacid (1.3 equiv) dropwise by syringe. The reaction mixture was warmed slowly to $25{ }^{\circ} \mathrm{C}$. The reaction mixture was then cooled to $0{ }^{\circ} \mathrm{C}$ and quenched with saturated $\mathrm{Na}_{2} \mathrm{CO}_{3}(2 \mathrm{~mL})$ and warmed to $25{ }^{\circ} \mathrm{C}$. The reaction mixture was diluted with EtOAc $(2 \mathrm{~mL})$ and $\mathrm{H}_{2} \mathrm{O}(2 \mathrm{~mL})$, the layers were separated, and the aqueous layer was extracted four times with EtOAc. The combined organic layers were washed once with brine $(5 \mathrm{~mL})$, dried over $\mathrm{Na}_{2} \mathrm{SO}_{4}$, filtered, and concentrated in vacuo. The diastereomeric ratio of products was determined by $\mathrm{GC}$ analysis of the unpurified product mixture and confirmed using ${ }^{1} \mathrm{H}$ NMR spectroscopy.

Procedure B: General Procedure for $\mathrm{Me}_{3} \mathrm{Al} / \mathrm{BF}_{3} \cdot \mathrm{OEt}_{2}$ Mediated Rearrangement of Vinyl Acetals. ${ }^{6}$ To a cooled $\left(-78{ }^{\circ} \mathrm{C}\right)$ solution of the vinyl acetal $(0.1-0.15 \mathrm{mmol})$ in $\mathrm{PhMe}(0.15 \mathrm{M})$ was added the $\mathrm{Me}_{3} \mathrm{Al}(4.0$ equiv, 2.0 M solution in hexanes) dropwise by syringe. The mixture was allowed to stir for $2 \mathrm{~min}$ at $-78^{\circ} \mathrm{C}$. At this time, $\mathrm{BF}_{3} \cdot \mathrm{OEt}_{2}$ (1.1 equiv) was added dropwise by syringe, and the reaction mixture was allowed to stir for $30 \mathrm{~min}$. Saturated $\mathrm{Na}_{2} \mathrm{CO}_{3}(2 \mathrm{~mL})$ was added and the reaction mixture was warmed to $25{ }^{\circ} \mathrm{C}$. The reaction mixture was diluted with EtOAc $(2 \mathrm{~mL})$ and $\mathrm{H}_{2} \mathrm{O}(2 \mathrm{~mL})$, the layers were separated, and the aqueous layer was extracted four times with EtOAc. The combined organic layers were washed once with brine $(5 \mathrm{~mL})$, dried over $\mathrm{Na}_{2} \mathrm{SO}_{4}$, filtered, and concentrated in vacuo. The diastereomeric ratio of products was determined by $\mathrm{GC}$ analysis of the unpurified product mixture and confirmed using ${ }^{1} \mathrm{H}$ NMR spectroscopy. 


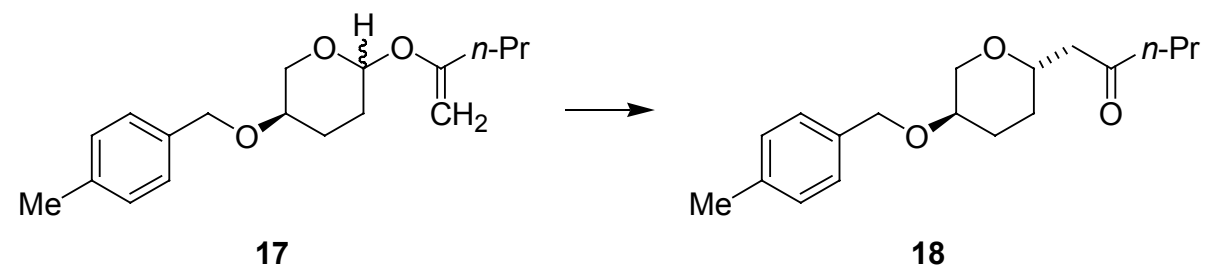

4-(4-Methylbenzyloxy)tetrahydropyran-1-(2'-n-propyl-2'-ethanone) (18). According to general procedure A, vinyl acetal $17(0.04 \mathrm{~g}, 0.12 \mathrm{mmol})$ in $\mathrm{PhMe}(0.9 \mathrm{~mL})$ was treated with $\mathrm{BF}_{3} \cdot \mathrm{OEt}_{2}(20.0 \mu \mathrm{L}, 0.16$ mmol). The reaction mixture was warmed to $25{ }^{\circ} \mathrm{C}$. After $1 \mathrm{~h}$ the reaction mixture was cooled to $0{ }^{\circ} \mathrm{C}$ and treated with a saturated aqueous solution of $\mathrm{Na}_{2} \mathrm{CO}_{3}(2 \mathrm{~mL})$. The slurry was then warmed to $25^{\circ} \mathrm{C}$ and diluted with EtOAc $(2 \mathrm{~mL})$ and $\mathrm{H}_{2} \mathrm{O}(2 \mathrm{~mL})$. The layers were separated and the aqueous layer was extracted with $3 \times$ $10 \mathrm{~mL}$ EtOAc. The combined organic layers were washed with brine $(10 \mathrm{~mL})$, dried over $\mathrm{Na}_{2} \mathrm{SO}_{4}$, filtered, and concentrated in vacuo to afford the product as a yellow oil as a 78:22 trans/cis mixture of isomers. ${ }^{7}$ The oil was purified by column chromatography $(0: 100-30: 70 \mathrm{EtOAc} /$ pentane $)$ to afford the product as a clear oil $(0.03 \mathrm{~g}$, $70 \%$ ): $\mathrm{GC} \mathrm{t}_{\mathrm{R}}$ (major) $15.6 \mathrm{~min}, \mathrm{t}_{\mathrm{R}}$ (minor) $13.3 \mathrm{~min}\left(80{ }^{\circ} \mathrm{C}\right.$ for $\left.1 \mathrm{~min}, 10^{\circ} / \mathrm{min}\right) ;{ }^{1} \mathrm{H} \mathrm{NMR}\left(400 \mathrm{MHz}, \mathrm{CDCl}_{3}\right) \delta$ 7.25-7.13 (m, 4H), $4.54(\mathrm{~d}, J=11.7,1 \mathrm{H}), 4.47(\mathrm{~d}, J=11.7,1 \mathrm{H}), 4.02$ (ddd, $J=10.7,4.8,2.3,1 \mathrm{H}), 3.72$ (dddd, $J=11.0,7.4,5.1,2.1,1 \mathrm{H}), 3.41(\mathrm{tt}, J=10.3,4.6,1 \mathrm{H}), 3.17(\mathrm{t}, J=10.5,1 \mathrm{H}), 2.63(\mathrm{dd}, J=15.8,7.6,1 \mathrm{H}), 2.48$ $2.35(\mathrm{~m}, 3 \mathrm{H}), 2.33(\mathrm{~s}, 3 \mathrm{H}), 2.21-2.12(\mathrm{~m}, 1 \mathrm{H}), 1.76(\mathrm{ddd}, J=13.2,5.7,3.3,1 \mathrm{H}), 1.60(\mathrm{q}, J=7.4,2 \mathrm{H}), 1.53-$ $1.42(\mathrm{~m}, \quad 1 \mathrm{H}), \quad 1.36-1.28 \quad(\mathrm{~m}, \quad 1 \mathrm{H}), 0.90 \quad(\mathrm{t}, \quad J=7.3, \quad 3 \mathrm{H}) ;{ }^{13} \mathrm{C} \quad \mathrm{NMR} \quad\left(125 \mathrm{MHz}, \mathrm{CDCl}_{3}\right)$ $\delta 209.5,137.8,135.9,129.5,128.2,74.2,73.0,71.2,71.1,49.0,46.1,31.0,30.4,21.6,17.4,14.1$; IR (thin film) 2923, 2856, 1730, 1452, 1268, 1023, $710 \mathrm{~cm}^{-1}$ HRMS (GC-MS, EI) $\mathrm{m} / z$ calcd for $\mathrm{C}_{18} \mathrm{H}_{26} \mathrm{O}_{3}(\mathrm{M}+\mathrm{Na})^{+}$ 313.1780, found 313.1778. Anal. Calcd for $\mathrm{C}_{18} \mathrm{H}_{26} \mathrm{O}_{3}: \mathrm{C}, 74.45 ; \mathrm{H}, 9.02$. Found: C, 74.38; H, 9.11.

Crossover Experiment using $\mathbf{B F}_{3} \cdot \mathbf{O E t}_{2}$. Using the general procedure $\mathbf{A}$, vinyl acetals $\mathbf{1 0}(0.025 \mathrm{~g}, 0.09 \mathrm{mmol})$ and $17(0.023 \mathrm{~g}, 0.09 \mathrm{mmol})$ in $\mathrm{PhMe}(0.5 \mathrm{~mL})$ were treated with $\mathrm{BF}_{3} \cdot \mathrm{OEt}_{2}(30.0 \mu \mathrm{L}, 0.24 \mathrm{mmol})$. Upon workup, the combined organic layers were filtered through a silica plug and concentrated in vacuo to afford the product mixture as a colorless oil.

Crossover Experiment using $\mathbf{M e}_{3} \mathbf{A} / \mathbf{B F}_{3} \cdot \mathbf{O E t}_{2}$. Using the general procedure $\mathbf{B}$, vinyl acetals $\mathbf{1 0}(0.018 \mathrm{~g}, 0.06$ $\mathrm{mmol})$ and $17(0.015 \mathrm{~g}, 0.06 \mathrm{mmol})$ in $\mathrm{PhMe}(0.4 \mathrm{~mL})$ were treated with $\mathrm{Me}_{3} \mathrm{Al}(0.24 \mathrm{~mL}, 2.0 \mathrm{M}$ solution in hexanes, $0.50 \mathrm{mmol})$ and $\mathrm{BF}_{3} \cdot \mathrm{OEt}_{2}(20.0 \mu \mathrm{L}, 0.15 \mathrm{mmol})$. Upon workup, the combined organic layers were filtered through a silica plug and concentrated in vacuo to afford the product mixture as a colorless oil

\section{Control Experiments for the Rearrangement Reactions of Vinyl Acetals with Other Lewis-Acids.}

Using the general procedure $\mathbf{A}$, vinyl acetal $\mathbf{1 0}$ was rearranged to ketone $\mathbf{1 2}$ using the Lewis acids $\mathrm{SnCl}_{4}, \mathrm{TiCl}_{4}$, and $\mathrm{Me}_{3} \mathrm{Si}$-OTf. GC analysis of the unpurified product mixture indicated that in all cases the rearrangement reaction proceeded to afford 12 with 80:20 (+/-3\%) 1,4-trans to 1,4-cis diastereoselectivity.

\section{Control Experiments for the Rearrangement Reaction of Vinyl Acetals in Other Solvents.}

Using the general procedure $\mathbf{B}$, vinyl acetal $\mathbf{1 0}$ was rearranged to ketone $\mathbf{1 2}$ using the solvents hexanes, $\mathrm{CH}_{2} \mathrm{Cl}_{2}$, and, nitropropane. GC analysis of the unpurified product mixture indicated that the rearrangement reaction proceeded to afford $\mathbf{1 2}$ with high 1,4-trans to 1,4-cis diastereoselectivity. The stereoselectivity ranged from 75:25 (trans:cis) in hexanes to 90:10 (trans:cis) in $\mathrm{CH}_{2} \mathrm{Cl}_{2}$ and nitropropane. 


\section{Stereochemical Proofs of Nucleophilic Substituted Products and Rearrangement Products}<smiles>CC(=O)C[C@H]1CC[C@@H](OCc2ccccc2)CO1</smiles>

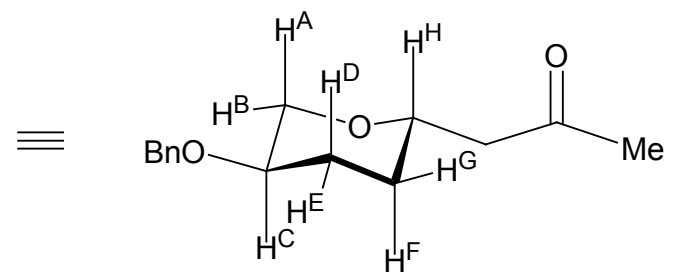

trans-4-Benzyloxytetrahydropyran-1-(2'-methyl-2'-ethanone) (12).

$\mathrm{H}^{\mathrm{A}}: \quad \delta 3.19, \mathrm{t}, J=10.5\left(\mathrm{gem}, \mathrm{ax}^{\mathrm{A}}-\mathrm{ax}^{\mathrm{C}}\right)$

$\mathrm{H}^{\mathrm{B}}: \quad \delta 4.05, \mathrm{ddd}, J=10.8(\mathrm{gem}), 4.7\left(\mathrm{eq}^{\mathrm{A}}-\mathrm{ax}^{\mathrm{C}}\right), 2.3\left(\mathrm{~W}-\right.$ coupling to $\left.\mathrm{H}^{\mathrm{E}}\right)$

$\mathrm{H}^{\mathrm{C}}: \quad \delta 3.44, \mathrm{tt}, J=10.3\left(\mathrm{ax}^{\mathrm{C}}-\mathrm{ax}^{\mathrm{A}}, \mathrm{ax}^{\mathrm{C}}-\mathrm{ax}^{\mathrm{D}}\right), 4.6\left(\mathrm{ax}^{\mathrm{C}}-\mathrm{eq}^{\mathrm{B}}, \mathrm{ax}^{\mathrm{C}}-\mathrm{eq}^{\mathrm{E}}\right)$

$\mathrm{H}^{\mathrm{F}}: \quad \delta 1.49, \mathrm{tdd}, J=13.2\left(\mathrm{gem}, \mathrm{ax}^{\mathrm{F}}-\mathrm{ax}^{\mathrm{D} / \mathrm{H}}\right), 10.9\left(\mathrm{ax}^{\mathrm{F}}-\mathrm{ax}^{\mathrm{D} / \mathrm{H}}\right), 4.1\left(\mathrm{ax}^{\mathrm{F}}-\mathrm{eq}^{\mathrm{E}}\right)$<smiles>C#CCCCCCC(=O)C[C@H]1CC[C@@H](OCc2ccccc2)CO1</smiles>

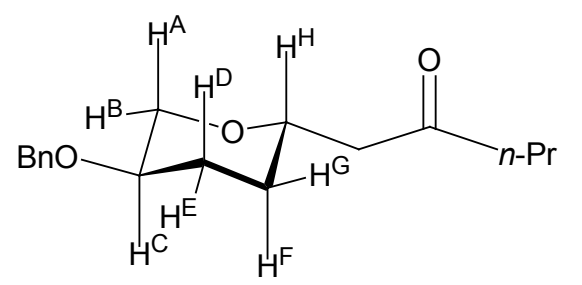

trans-4-Benzyloxytetrahydropyran-1-(2'-n-propyl-2'-ethanone) (19).

$\mathrm{H}^{\mathrm{A}}: \quad \delta 3.19, \mathrm{t}, J=10.5\left(\mathrm{gem}, \mathrm{ax}^{\mathrm{A}}-\mathrm{ax}^{\mathrm{C}}\right)$

$\mathrm{H}^{\mathrm{B}}: \quad \delta 4.04, \mathrm{ddd}, J=10.8($ gem $), 4.7\left(\mathrm{eq}^{\mathrm{A}}-\mathrm{ax}^{\mathrm{C}}\right), 2.3\left(\mathrm{~W}-\right.$ coupling to $\left.\mathrm{H}^{\mathrm{E}}\right)$

$\mathrm{H}^{\mathrm{C}}: \quad \delta 3.43, \mathrm{tt}, J=10.3\left(\mathrm{ax}^{\mathrm{C}}-\mathrm{ax}^{\mathrm{A}}, \mathrm{ax}^{\mathrm{C}}-\mathrm{ax}^{\mathrm{D}}\right), 4.6\left(\mathrm{ax}^{\mathrm{C}}-\mathrm{eq}^{\mathrm{B}}, \mathrm{ax}^{\mathrm{C}}-\mathrm{eq}^{\mathrm{E}}\right)$

$\mathrm{H}^{\mathrm{F}}: \quad \delta 1.49, \mathrm{tdd}, J=13.1\left(\mathrm{gem}, \mathrm{ax}^{\mathrm{F}}-\mathrm{ax}^{\mathrm{D} / \mathrm{H}}\right), 11.0\left(\mathrm{ax}^{\mathrm{F}}-\mathrm{ax}^{\mathrm{D} / \mathrm{H}}\right), 4.2\left(\mathrm{ax}^{\mathrm{F}}-\mathrm{eq}^{\mathrm{E}}\right)$<smiles>CC(=O)C[C@H]1CC[C@@H](OCc2ccc(C)cc2)CO1</smiles>

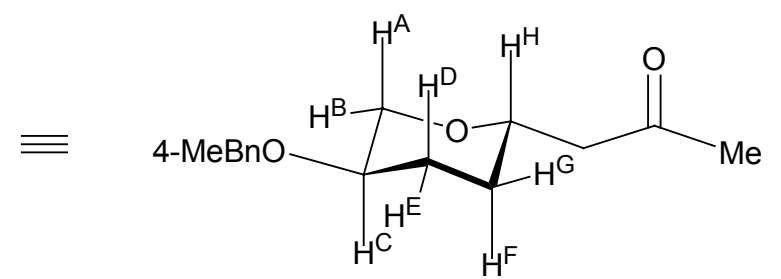

trans-4-(4-Methylbenzyloxy)tetrahydropyran-1-(2'-methyl-2'-ethanone) (20).

$\mathrm{H}^{\mathrm{A}}: \quad \delta 3.19, \mathrm{t}, J=10.5\left(\mathrm{gem}, \mathrm{ax}^{\mathrm{A}}-\mathrm{ax}^{\mathrm{C}}\right)$

$\mathrm{H}^{\mathrm{B}}: \quad \delta 4.02, \mathrm{ddd}, J=10.8(\mathrm{gem}), 4.7\left(\mathrm{eq}^{\mathrm{A}}-\mathrm{ax}^{\mathrm{C}}\right), 2.3\left(\mathrm{~W}-\right.$ coupling to $\left.\mathrm{H}^{\mathrm{E}}\right)$

$\mathrm{H}^{\mathrm{C}}: \quad \delta 3.42, \mathrm{tt}, J=10.3\left(\mathrm{ax}^{\mathrm{C}}-\mathrm{ax}^{\mathrm{A}}, \mathrm{ax}^{\mathrm{C}}-\mathrm{ax}^{\mathrm{D}}\right), 4.6\left(\mathrm{ax}^{\mathrm{C}}-\mathrm{eq}^{\mathrm{B}}, \mathrm{ax}^{\mathrm{C}}-\mathrm{eq}^{\mathrm{E}}\right)$

$\mathrm{H}^{\mathrm{F}}: \quad \delta 1.47, \mathrm{tdd}, J=13.2\left(\mathrm{gem}, \mathrm{ax}^{\mathrm{F}}-\mathrm{ax}^{\mathrm{D} / \mathrm{H}}\right), 10.9\left(\mathrm{ax}^{\mathrm{F}}-\mathrm{ax}^{\mathrm{D} / \mathrm{H}}\right), 4.1\left(\mathrm{ax}^{\mathrm{F}}-\mathrm{eq}^{\mathrm{E}}\right)$ 


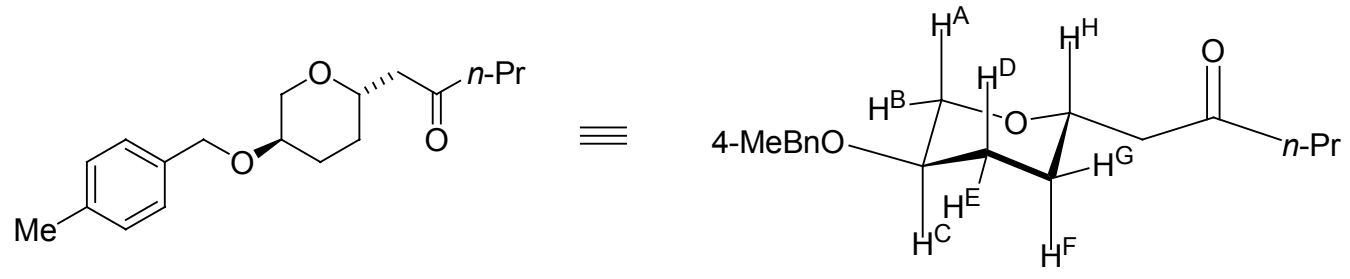

trans-4-(4-Methylbenzyloxy)tetrahydropyran-1-(2'-n-propyl-2'-ethanone) (18).

$$
\begin{array}{ll}
\mathrm{H}^{\mathrm{A}}: & \delta 3.17, \mathrm{t}, J=10.5\left(\mathrm{gem}, \mathrm{ax}^{\mathrm{A}}-\mathrm{ax}^{\mathrm{C}}\right) \\
\mathrm{H}^{\mathrm{B}}: & \delta 4.02, \mathrm{ddd}, J=10.7(\mathrm{gem}), 4.8\left(\mathrm{eq}^{\mathrm{A}}-\mathrm{ax}^{\mathrm{C}}\right), 2.3\left(\mathrm{~W}-\mathrm{coupling}_{\mathrm{C}} \mathrm{H}^{\mathrm{E}}\right) \\
\mathrm{H}^{\mathrm{C}}: & \delta 3.41, \mathrm{tt}, J=10.3\left(\mathrm{ax}^{\mathrm{C}}-\mathrm{ax}^{\mathrm{A}}, \mathrm{ax}^{\mathrm{C}}-\mathrm{ax}^{\mathrm{D}}\right), 4.6\left(\mathrm{ax}^{\mathrm{C}}-\mathrm{eq}^{\mathrm{B}}, \mathrm{ax}^{\mathrm{C}}-\mathrm{eq}^{\mathrm{E}}\right)
\end{array}
$$

\section{Bibliography}

(1)Pangborn, A. B.; Giardello, M. A.; Grubbs, R. H.; Rosen, R. K.; Timmers, F. J. Organometallics 1996, 15, 1518-1520.

1985, 50, 1582-1589.

(2)Brown, H. C.; Vara Prasad, J. V. N.; Zee, S.-H. J. Org. Chem.

(3)Anomeric ratio determined using ${ }^{1} \mathrm{H}$ NMR spectroscopy.

(4)Dimethyltitanocene was prepared from methyllithium and titanocene dichloride according to the procedure outlined in: Petasis, N. A.; Bzowej, E. I. Method for Olefination of Carbonyl Compounds Using Titanocene Derivatives. U.S. Patent 5,087,790, June 12, 1991.

(5)Corey E. J.; Gross, A. W. Tetrahedron Lett. 1984, 25, 495-498.

Soc. 2002, 124, 9720-9721.

(6)Zhang, Y.; Reynolds, N. T.; Manju, K.; Rovis, T. J. Am. Chem.

(7)Product diastereoselectivities determined by GC analysis of the unpurified reaction mixture and confirmed by ${ }^{1} \mathrm{H}$ NMR spectroscopy. 


\section{Analytical data}

A. GC traces and ${ }^{1} \mathrm{H}$ NMR spectra of unpurified isomer ratios:
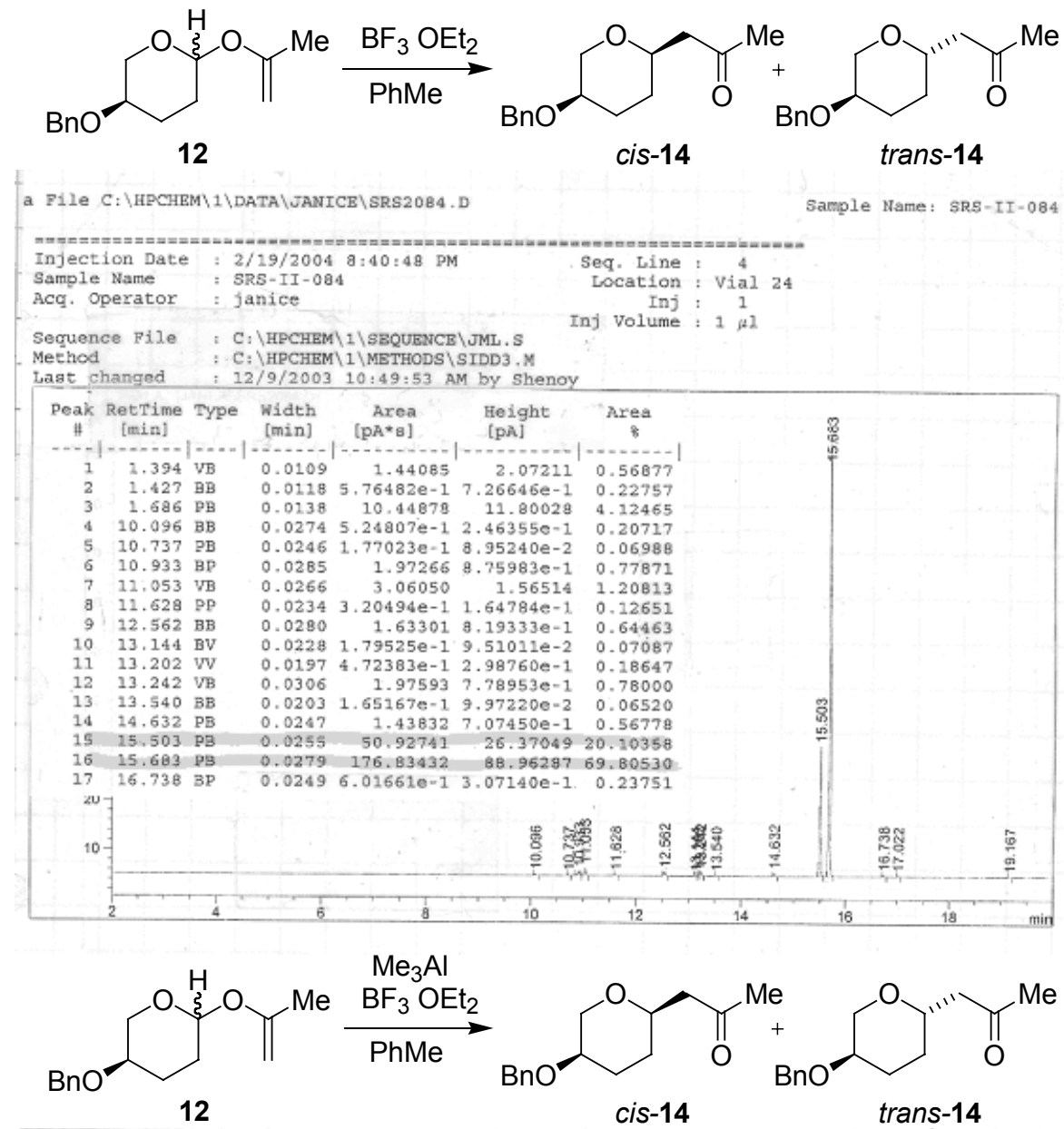

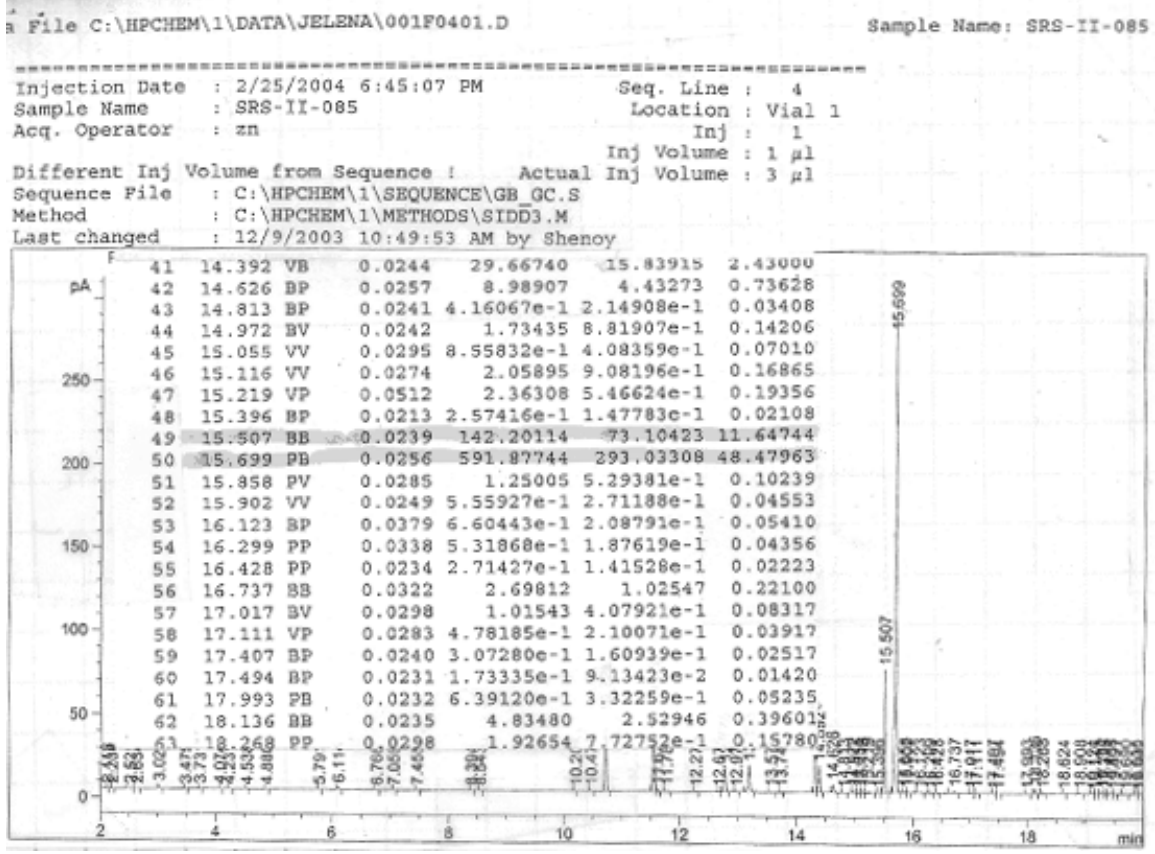




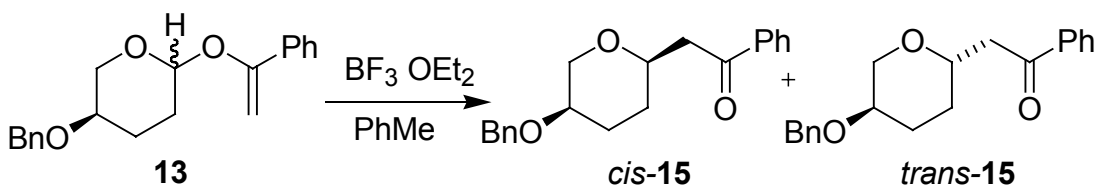

a File C:\APCHEM $1 \backslash$ DATA JSELENA\SRSII091.D

Sample Name: SRS-II-093

Crude SR8-1I-091
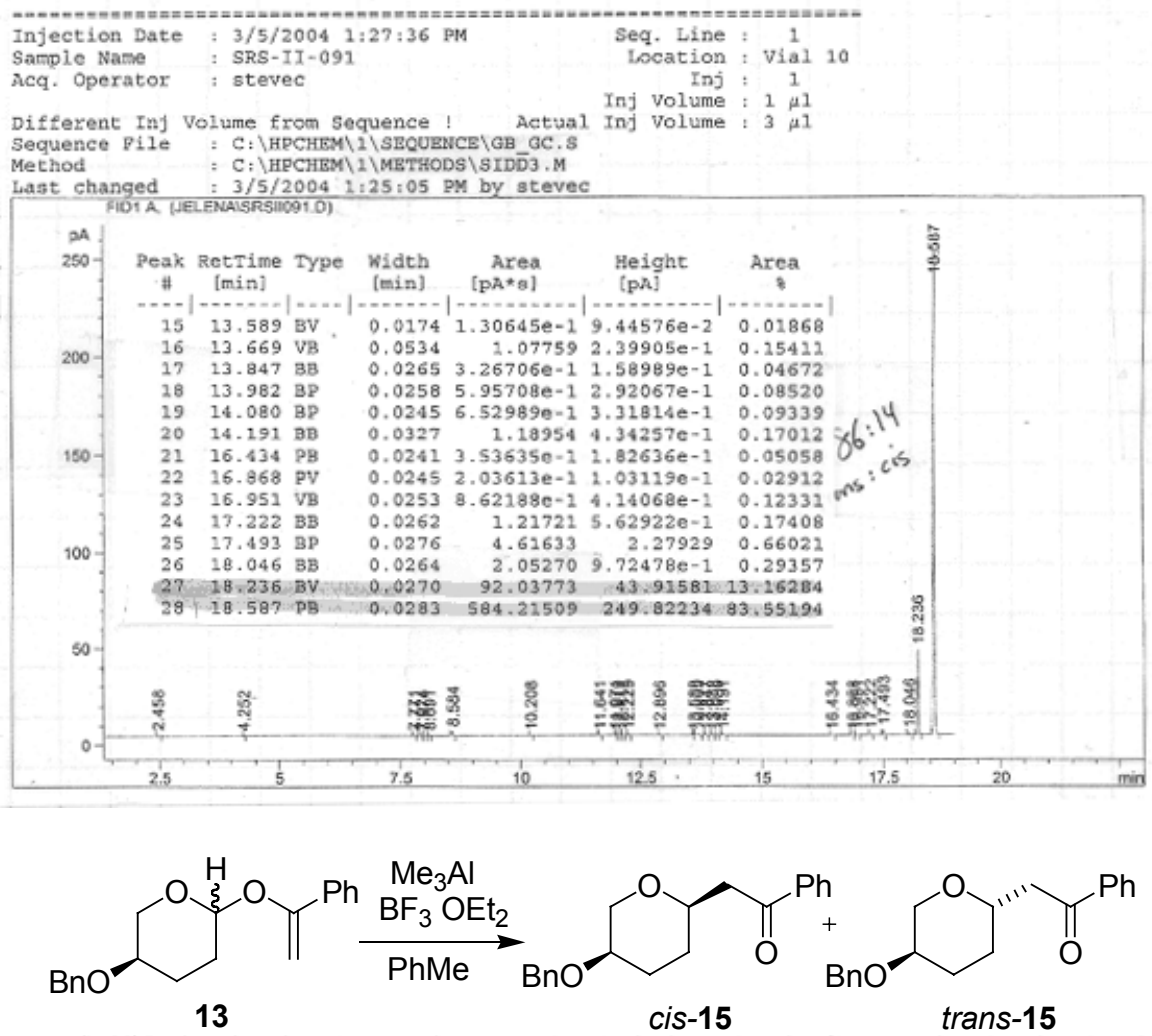

a File C: $\backslash$ HPCHEM $1 \backslash D A T A \backslash S R S \backslash S R S 2092 . D$

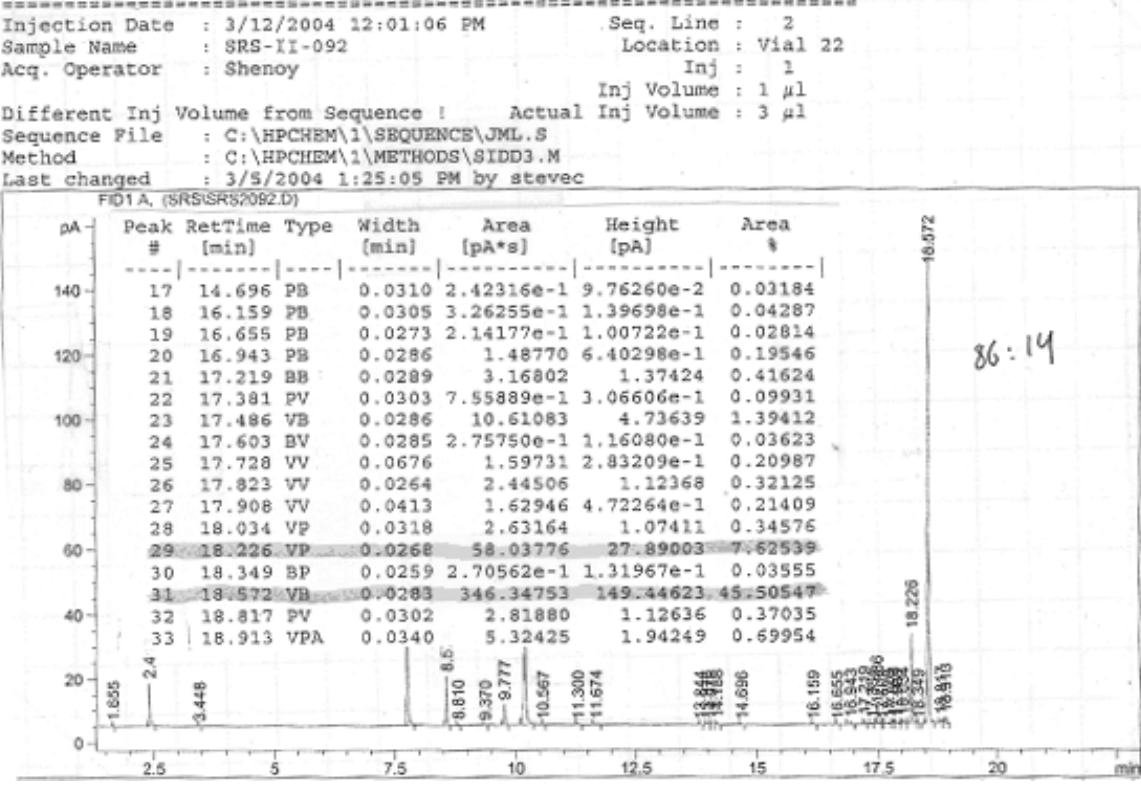




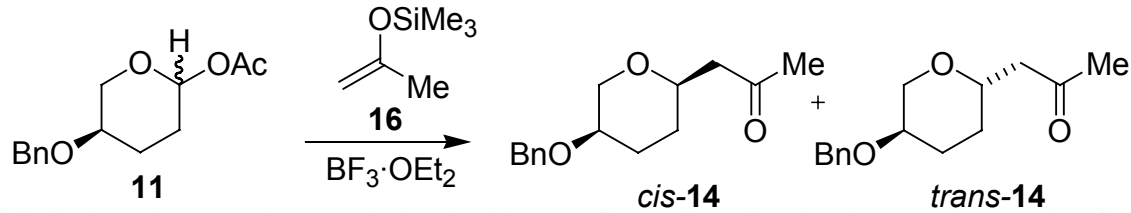

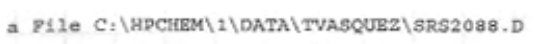

\begin{tabular}{|c|c|c|c|c|}
\hline $\begin{array}{l}\text { njection Date } \\
\text { ample Mame }\end{array}$ & $\begin{array}{l}: 2 / 26 / 2004 \quad 22: 51: 05 \\
\text { SRS-IT-088 }\end{array}$ & PM & seq. Line & : \\
\hline eq. Operacor & $\begin{array}{l}\text { : SRS-11-088 } \\
: \text { srs }\end{array}$ & & tocation & a vial \\
\hline & & Actual & $\begin{array}{l}\text { Inj Volume } \\
\text { Inj Volume }\end{array}$ & $\begin{array}{l}1 \\
1 \\
3 \\
3\end{array} \mu_{1}$ \\
\hline
\end{tabular}

Different Inj volume from Sequence , Actual Inj Volumo:

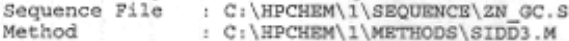

$\begin{array}{ll}\text { Method } & \text { : C: } \backslash \text { HPCHEM } 1 \backslash \text { MRTHODS } \backslash \text { SIDD } 3 . M \\ \text { Lase changed } & : 12 / 9 / 2003 \quad 10: 49+53 \text { AM by shenoy }\end{array}$

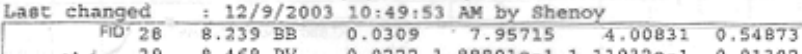

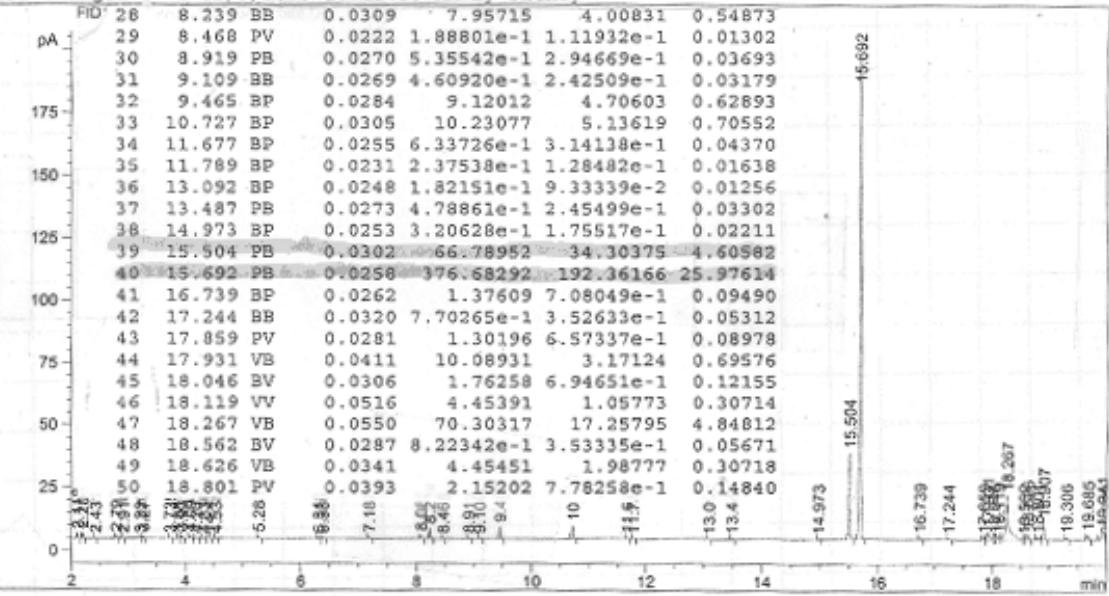<smiles>CC(=O)OC1CCC(OBr)CO1</smiles>

11

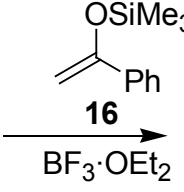

16

a File C: \HPCHEM $\backslash \backslash$ DATA \SRS\SRS2093.D<smiles>O=C(CC1CC[C@@H](OCc2ccccc2)CO1)Pc1ccccc1</smiles>

cis-15

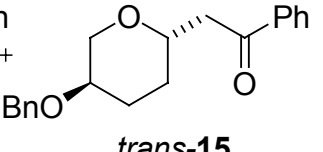

Sample Name: SRS-II-093

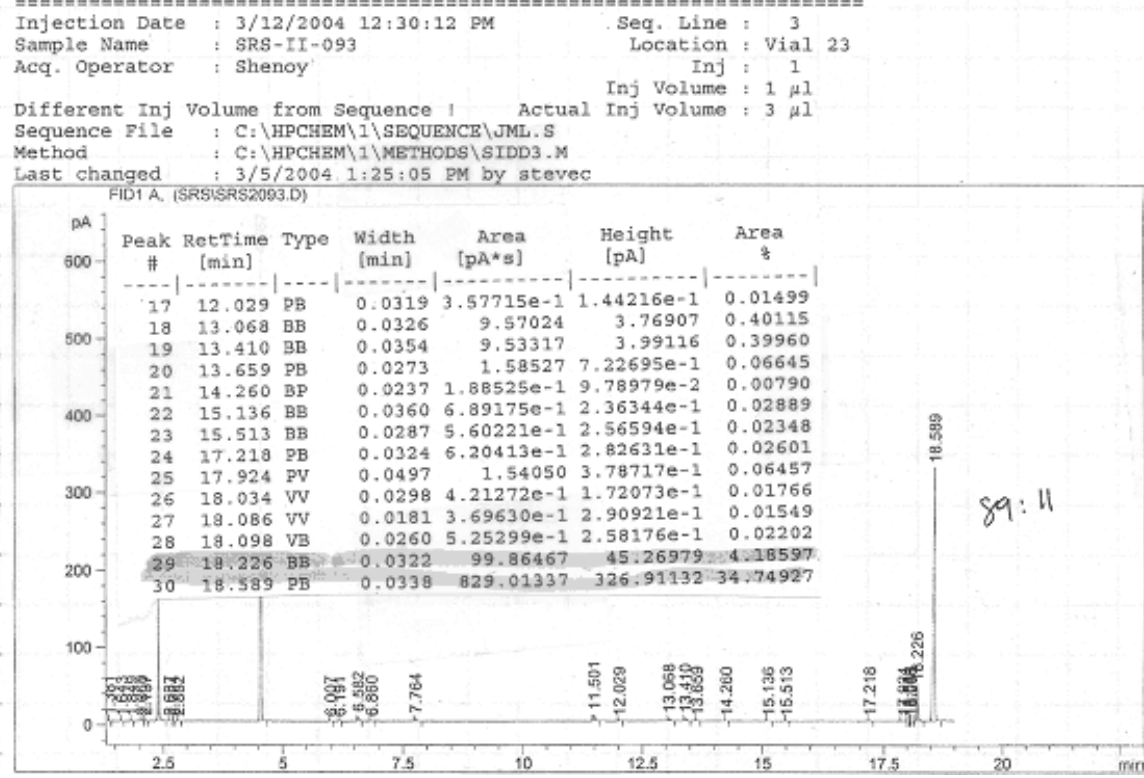


<smiles>C=C(C)O[C@H]1CC[C@@H](OCc2ccccc2)CO1</smiles>

12<smiles>CC(=O)C[C@H]1CC[C@@H](OCc2ccccc2)CO1</smiles>

14<smiles>CCCCC(=O)C[C@H]1CC[C@@H](OCc2ccccc2)CO1</smiles>

19

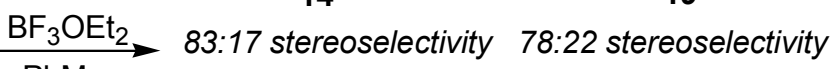
$\mathrm{PhMe}$<smiles>C=C(OCCC)OC1CCC(OC(C)C)CO1</smiles>

17<smiles>CCCCC(=O)CC1CCC(O[14CH3])CO1</smiles>

18

20

78:22 stereoselectivity $82: 18$ stereoselectivity

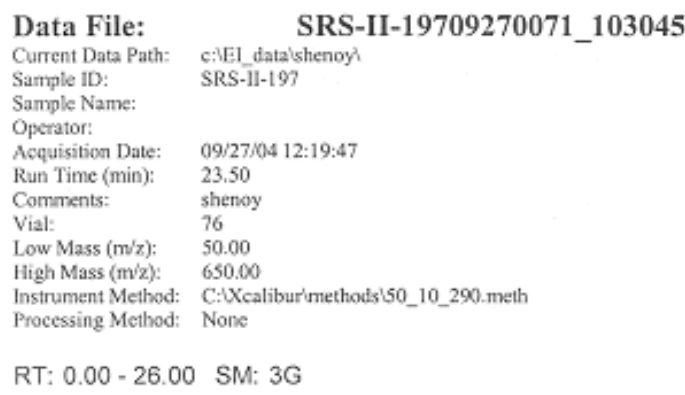

RT: $0.00-26.00$ SM: $3 G$

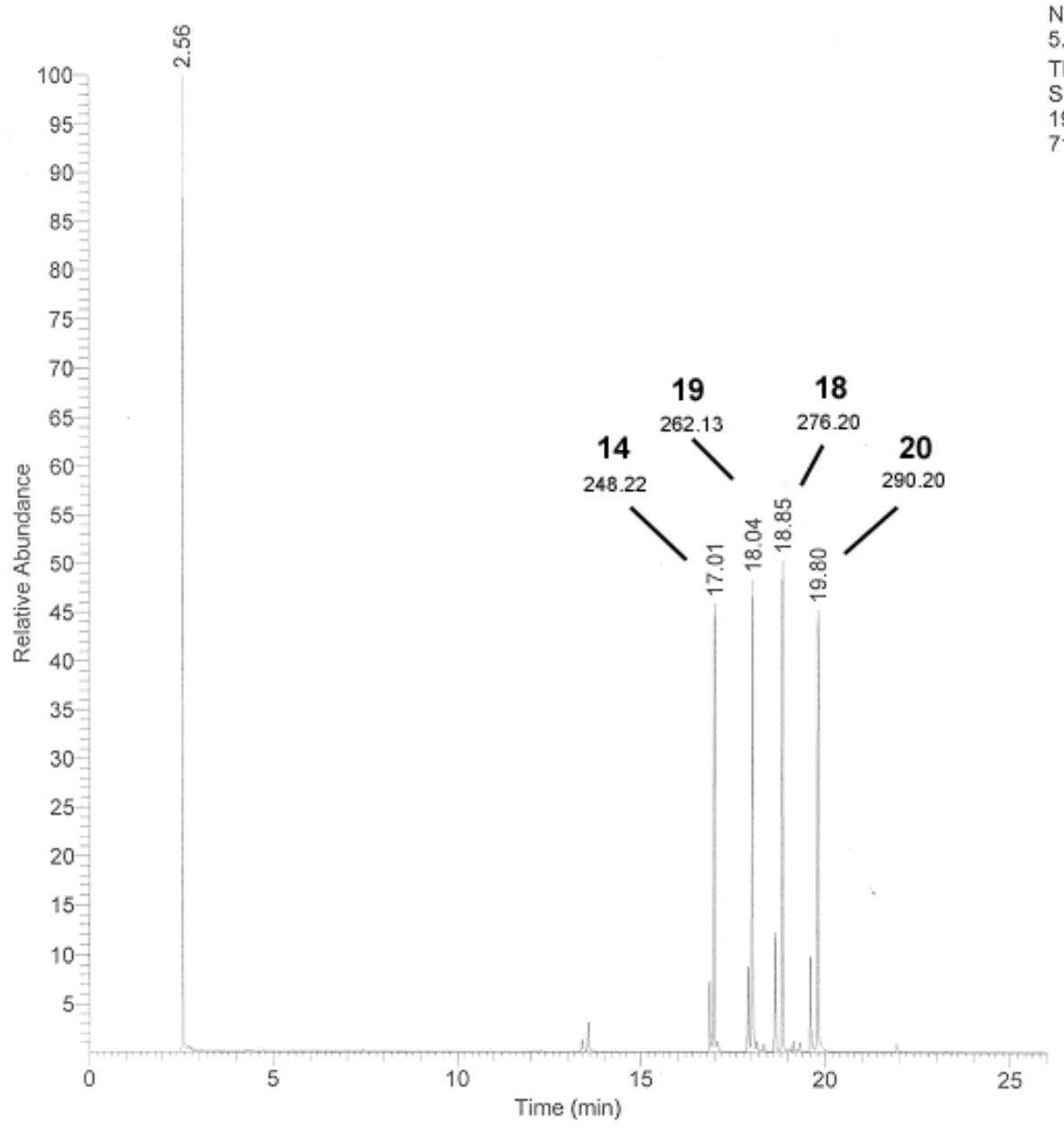

NL: 5.97E8 TIC MS SRS-11 197092700 $71+103045$ 
<smiles>C=C(C)O[C@H]1CC[C@@H](OCc2ccccc2)CO1</smiles>

12<smiles>CC(=O)C[C@H]1CC[C@@H](OCc2ccccc2)CO1</smiles>

14<smiles>CCCCC(=O)C[C@H]1CC[C@@H](OCc2ccccc2)CO1</smiles>

19

85:15 stereoselectivity $85: 15$ stereoselectivity<smiles>C=C(OCCC)O[C@H]1CC[C@@H](O[AlH2])CO1</smiles>

17<smiles>CCCCC(=O)CC1CC[C@@H](O[Al])CO1</smiles>

18

20

81:19 stereoselectivity $84: 16$ stereoselectivity

UCI MS Facility

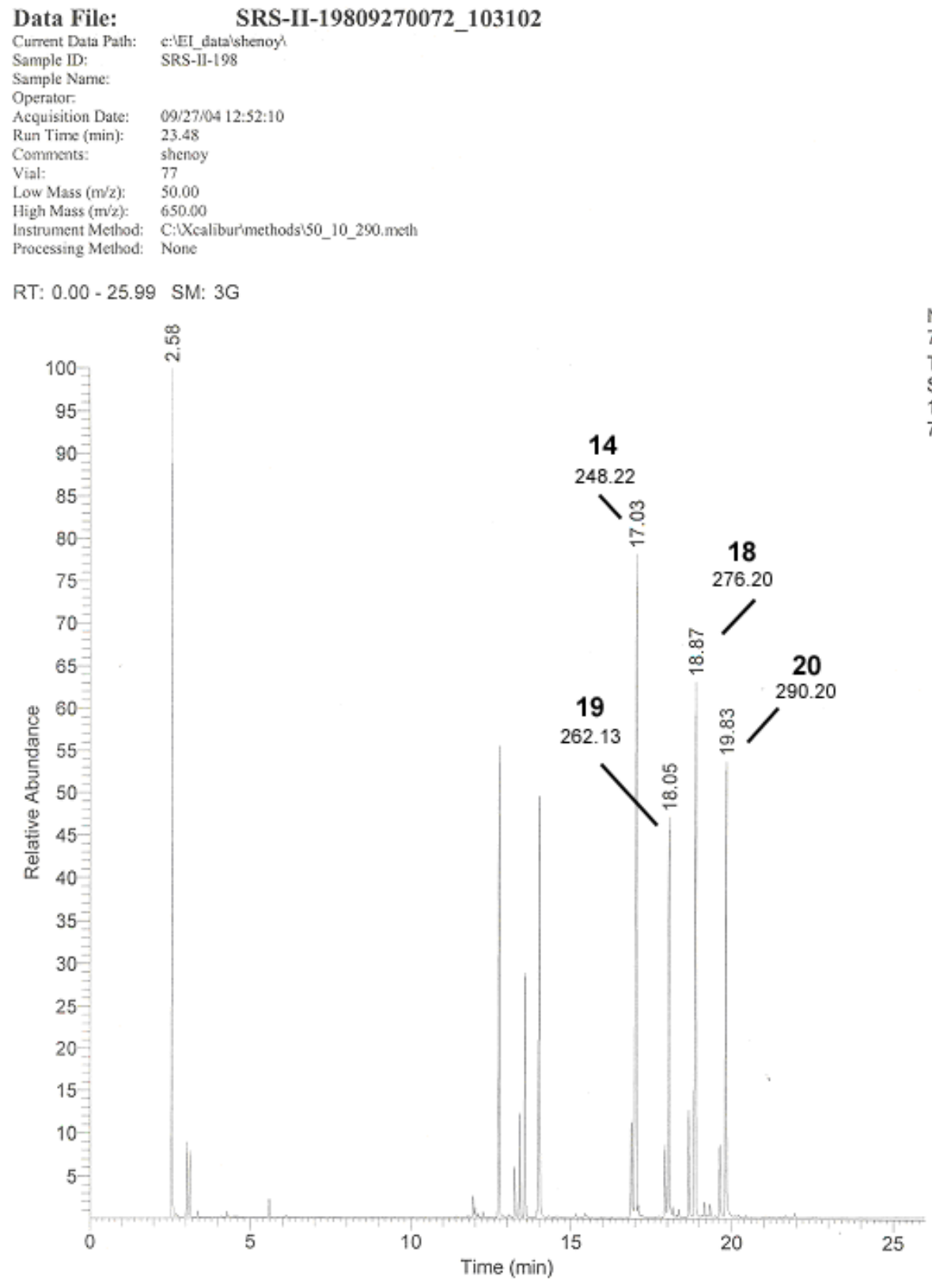

NL: 7.21E8

TIC MS SRS-II198092700 72_103102 
Selected ${ }^{1} \mathrm{H}$ NMR Spectra:
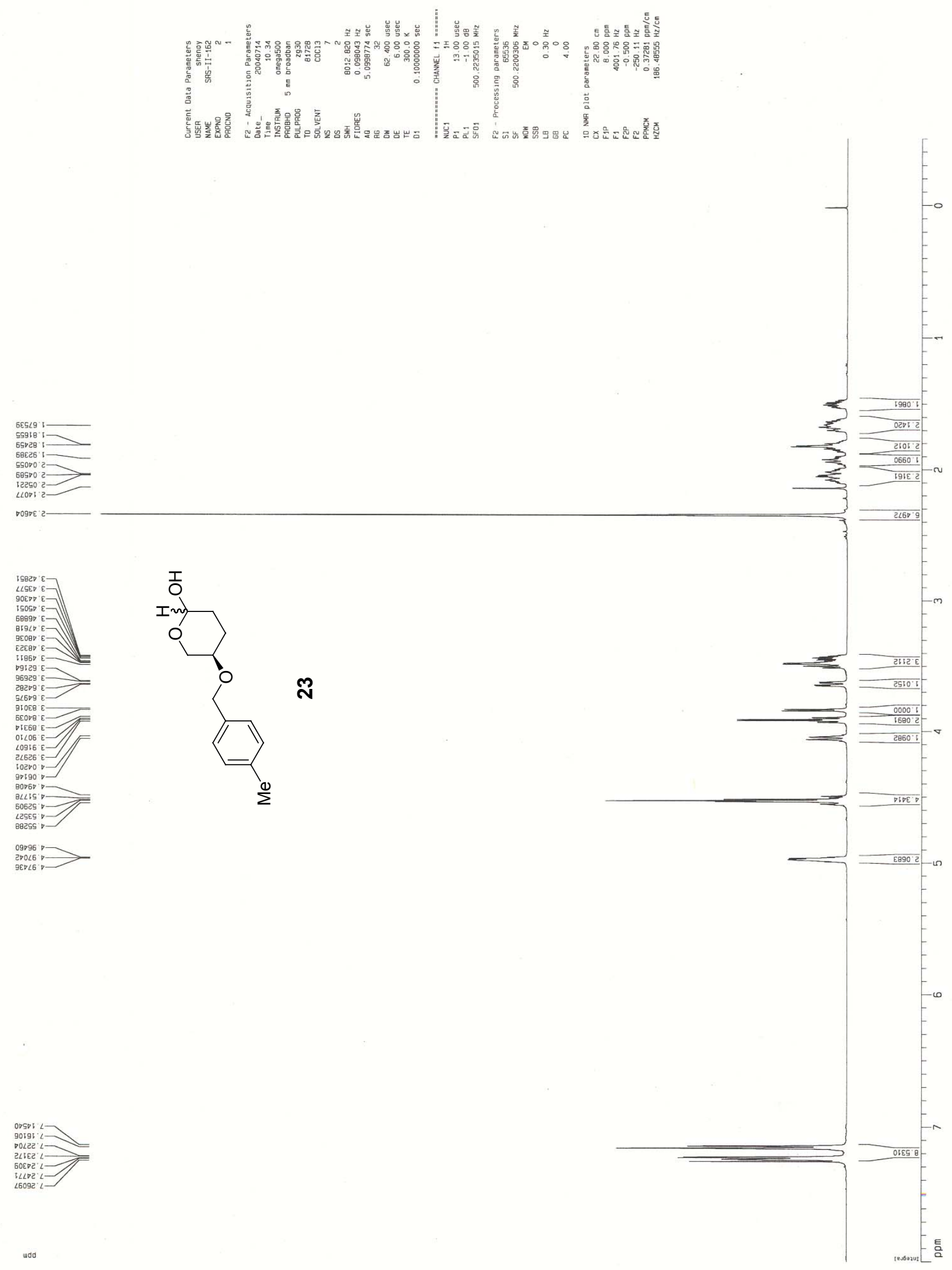

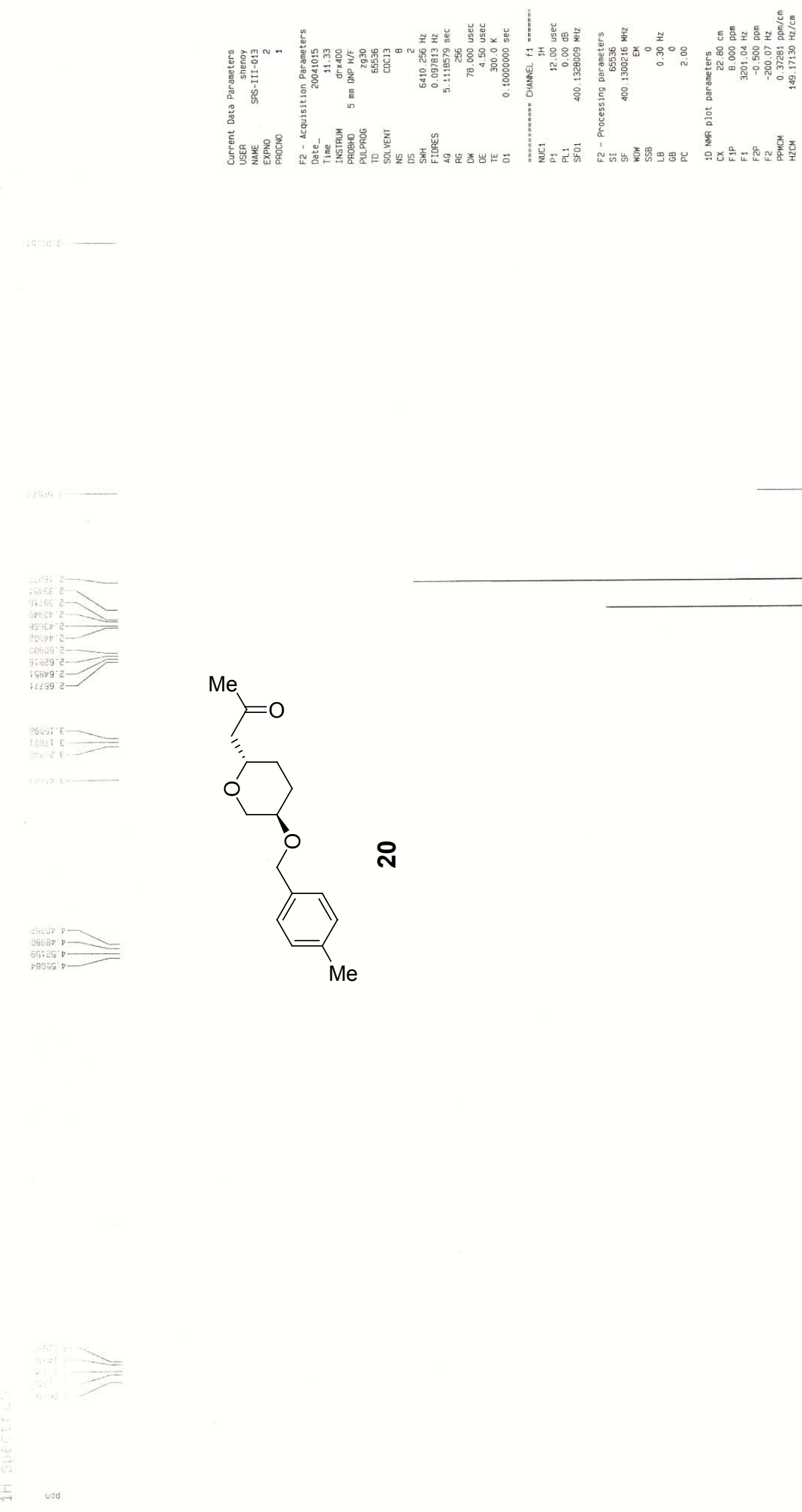

ำ

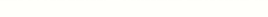




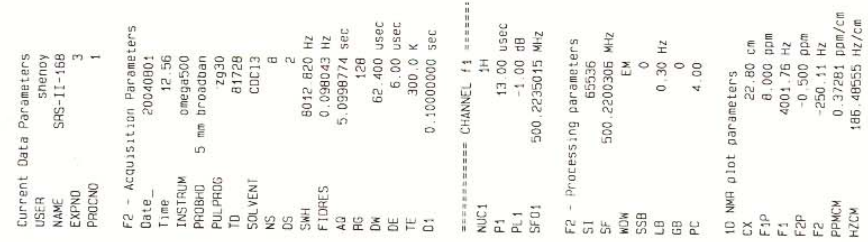

$8000 \cdot 0-$

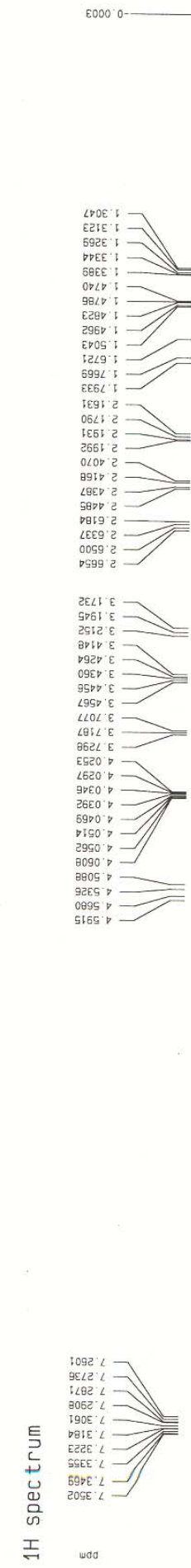<smiles>O=C1CCCC1</smiles>

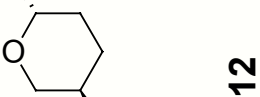

O

$\sim$
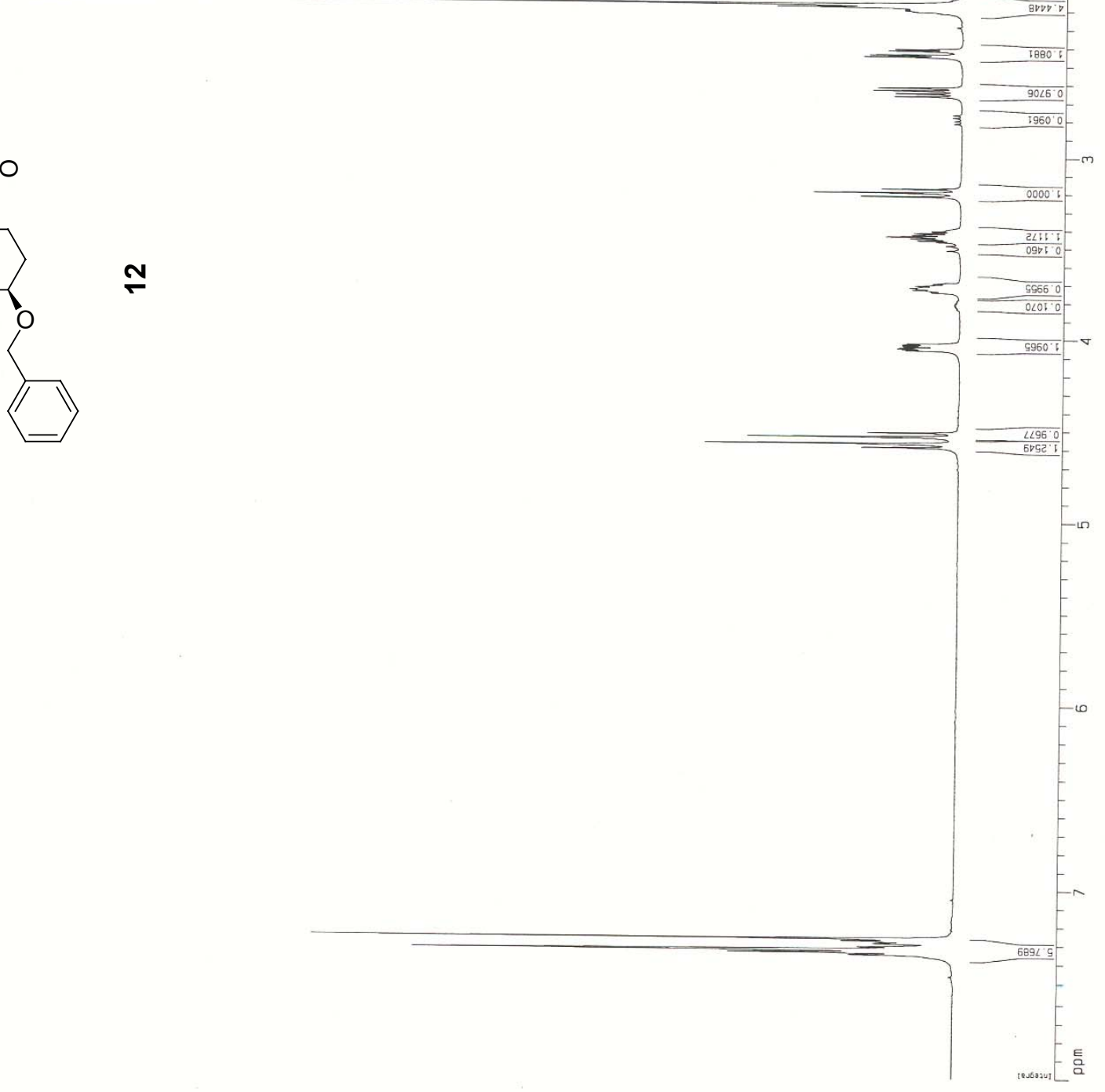


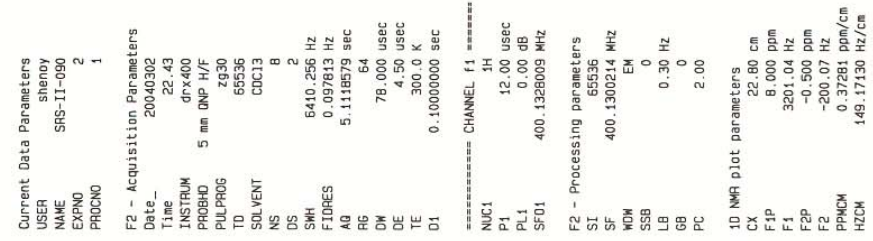

टहटฑ $0^{\circ}$

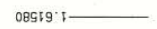

12980.57

5 เE60

$91900^{\prime} \cdot 5-2$
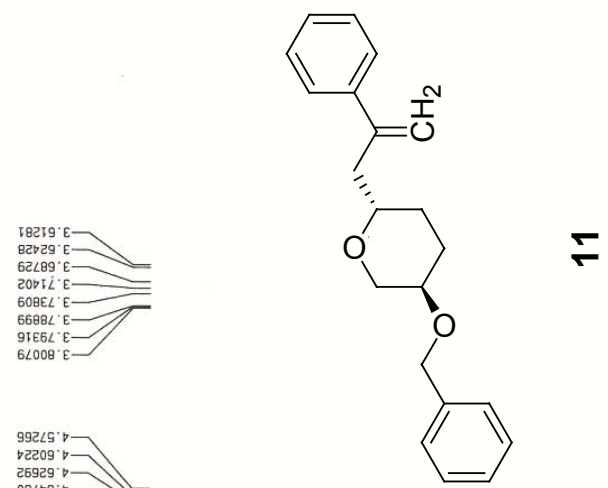

g9z $\angle g^{\prime}, r$

252209"

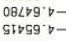

$\angle 9669^{\circ} p$

69698 ' $\square$

992Et.

costr

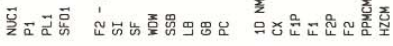

$F$
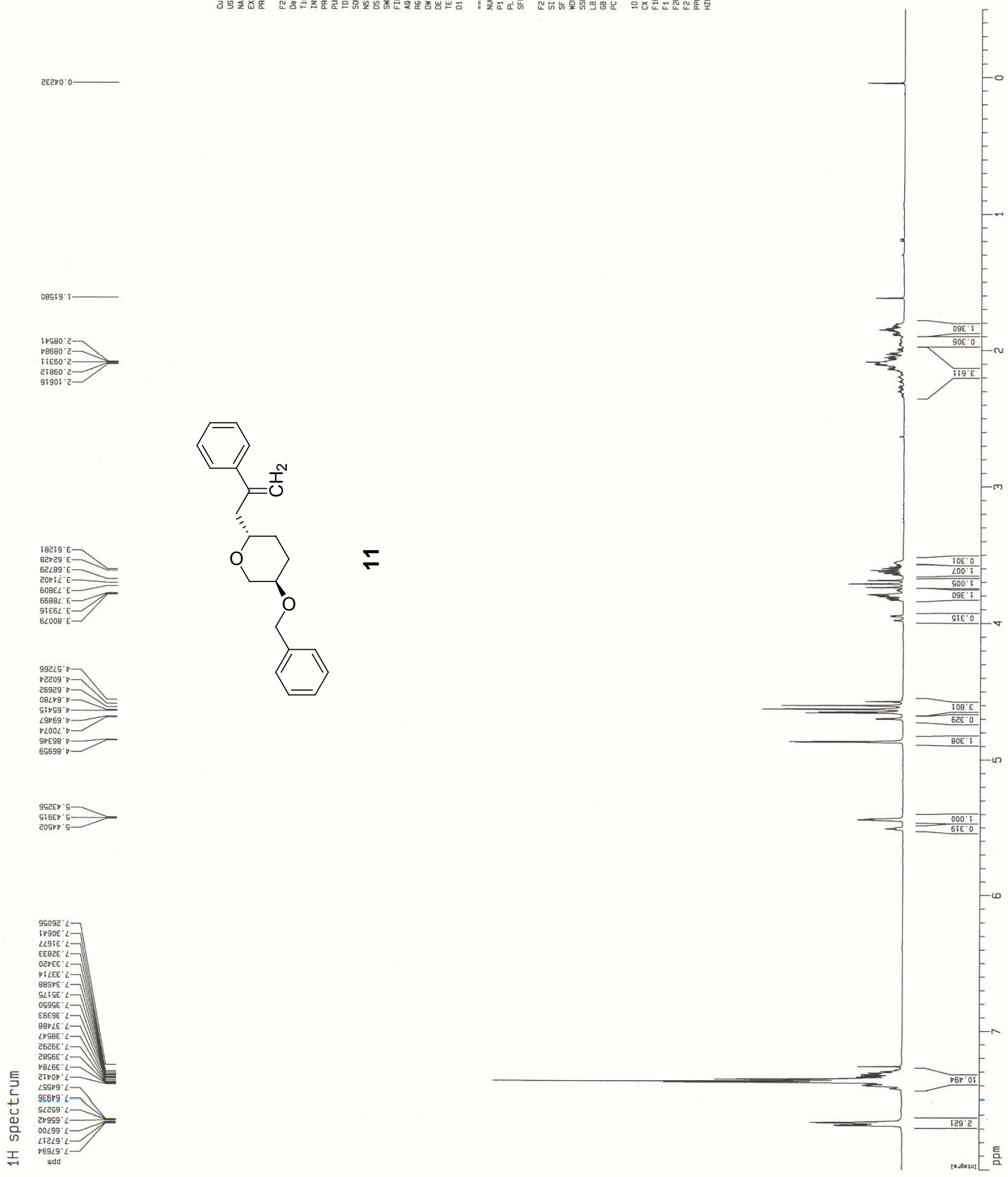

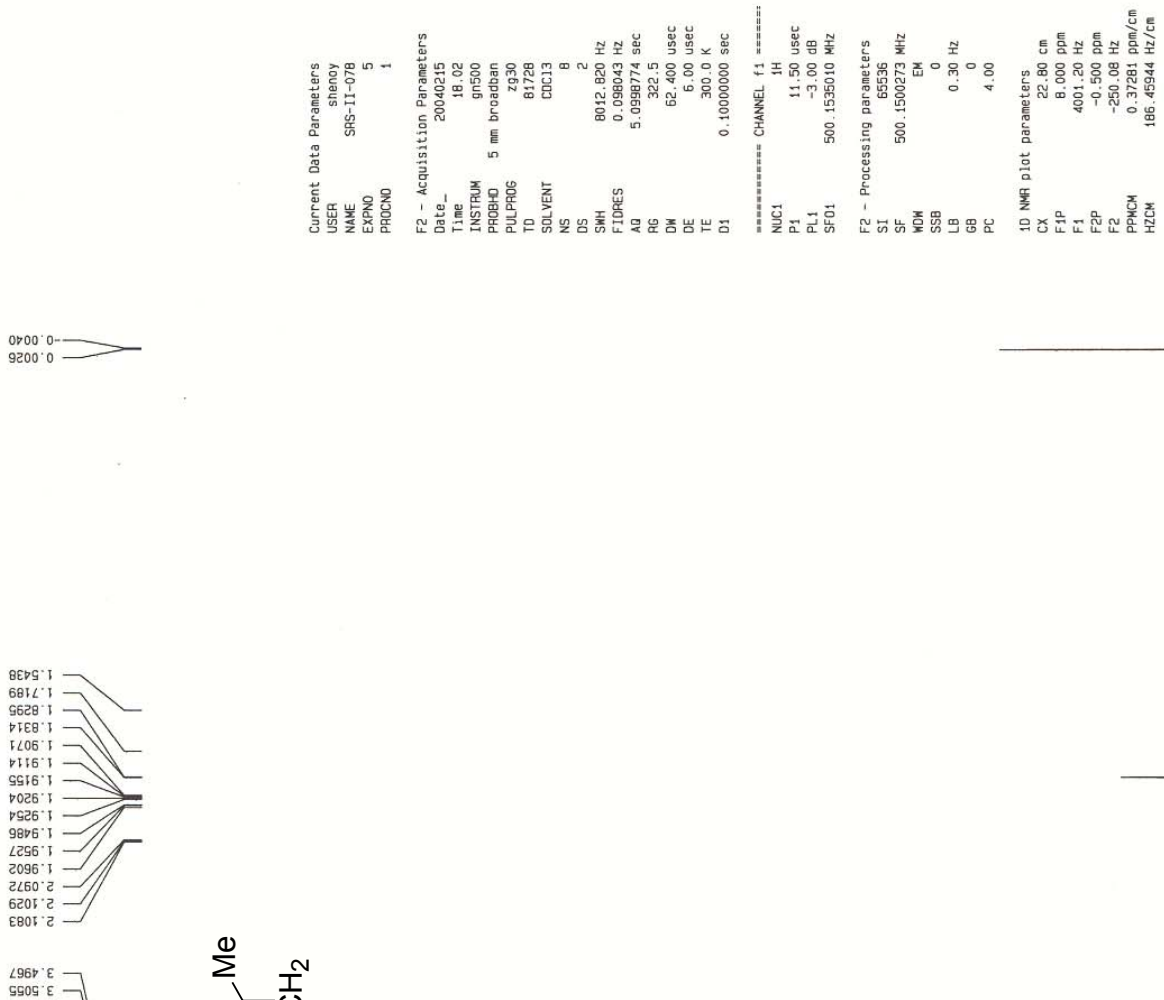

$\angle t T S \cdot \varepsilon$

$\begin{aligned} & \text { THCS } \varepsilon \\ & \text { टEES } \varepsilon\end{aligned}$

เB6s $\varepsilon]$

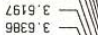

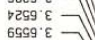

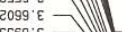

$\varepsilon 566^{\circ} \varepsilon-1$

${ }^{\circ} \mathrm{coc} \varepsilon$

tot:

sese:

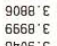

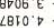

$\angle 2200^{\circ}$,

$\$ 200^{\circ} \cdot \mathrm{C}$

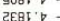

$602 e^{\prime \prime}$

seps' $\Rightarrow$

Coss' $\Rightarrow$

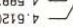

sore

1952.2

$8002 \cdot 9$

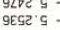

$\sum_{=}^{N} \stackrel{N}{U}^{N}$

O

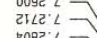

poobe' $\angle 2$

8662.2

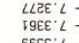

\section{우}
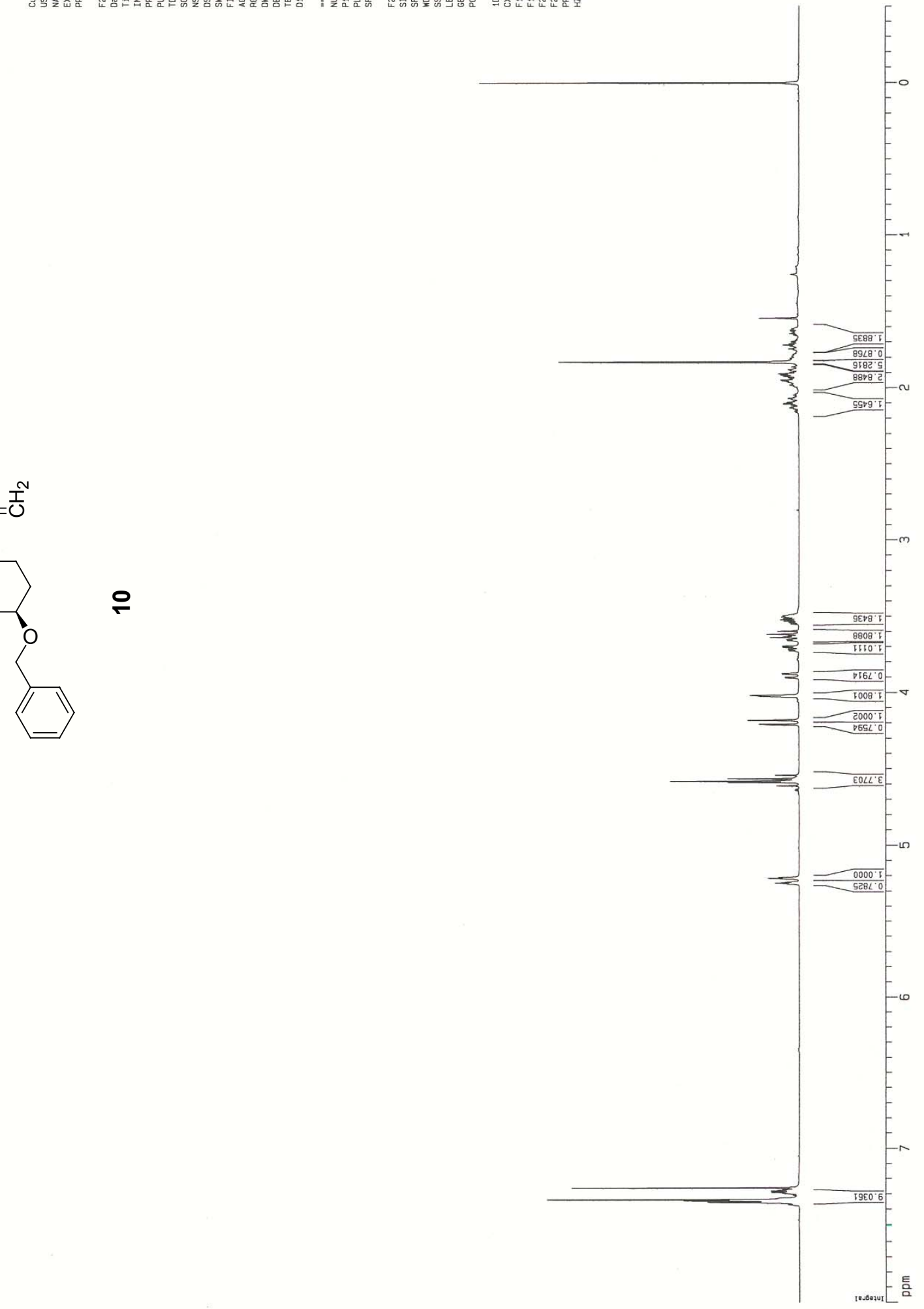